ARTICLE

https://doi.org/10.1038/s41467-020-16526-9

\title{
Triggering typical nemaline myopathy with compound heterozygous nebulin mutations reveals myofilament structural changes as pathomechanism
}

Johan Lindqvist ${ }^{1}$, Weikang Ma (iD ${ }^{2}$, Frank Li ${ }^{1}$, Yaeren Hernandez ${ }^{1}$, Justin Kolb ${ }^{1}$, Balazs Kiss ${ }^{1}$, Paola Tonino ${ }^{1}$, Robbert van der Pijl ${ }^{1}$, Esmat Karimi ${ }^{1}$, Henry Gong ${ }^{2}$, Josh Strom ${ }^{1}$, Zaynab Hourani ${ }^{1}$, John E. Smith III (D) ${ }^{1}$, Coen Ottenheijm¹, Thomas Irving ${ }^{2}$ \& Henk Granzier (1) 1,3凶

Nebulin is a giant protein that winds around the actin filaments in the skeletal muscle sarcomere. Compound-heterozygous mutations in the nebulin gene (NEB) cause typical nemaline myopathy (NM), a muscle disorder characterized by muscle weakness with limited treatment options. We created a mouse model with a missense mutation p.Ser6366lle and a deletion of NEB exon 55, the Compound-Het model that resembles typical NM. We show that Compound-Het mice are growth-retarded and have muscle weakness. Muscles have a reduced myofibrillar fractional-area and sarcomeres are disorganized, contain rod bodies, and have longer thin filaments. In contrast to nebulin-based severe NM where haplo-insufficiency is the disease driver, Compound-Het mice express normal amounts of nebulin. X-ray diffraction revealed that the actin filament is twisted with a larger radius, that tropomyosin and troponin behavior is altered, and that the myofilament spacing is increased. The unique disease mechanism of nebulin-based typical NM reveals novel therapeutic targets.

\footnotetext{
${ }^{1}$ Department of Cellular and Molecular Medicine, University of Arizona, 1656 East Mabel Street, Tucson, AZ 85724-5217, USA. ${ }^{2}$ Department of Biology, Illinois Institute of Technology, Chicago, IL 60616, USA. ${ }^{3}$ Sarver Molecular Cardiovascular Research Program, University of Arizona, Tucson, AZ 85721, USA.

凶email: granzier@email.arizona.edu
} 
$\mathrm{N}$ emaline myopathy (NM) is a heterogeneous disease with varying age of onset and severity and is characterized by skeletal muscle weakness, muscle atrophy, and intracellular nemaline rod bodies ${ }^{1-5}$. It can clinically be divided into subtypes of which the typical subtype (congenital and static or only slowly progressive) accounts for about half of the patients ${ }^{5-8}$. The most common cause of typical NM is mutations in the NEB gene that encodes the sarcomeric protein nebulin ${ }^{3,9,10}$. Nebulin is a $\sim 800 \mathrm{kDa}$ actin-binding protein that winds around the actin filament, with its C-terminus anchored in the Z-disk and its $\mathrm{N}$ terminus near the thin filament pointed-end ${ }^{11-18}$. The structure of nebulin is highly repetitive with 35 amino acid modules that bind actin and that form super-repeats ${ }^{11-14,19,20}$. Knockout mice have revealed nebulin's importance for thin-filament length regulation and stability, force production (altered cross-bridge cycling kinetics and calcium sensitivity) and alignment of myofibrils ${ }^{21-33}$.

Over 240 disease-causing mutations have been identified in $N E B$; the majority of patients are compound-heterozygous 9,34 . A missense mutation needs to be combined with a more disruptive mutation, such as a nonsense mutation, to cause typical $\mathrm{NM}^{9}$. The mechanisms by which mutations result in muscle weakness and atrophy are unclear. Current animal models with genetically modified nebulins fail to mimic typical NM patients due to, for example, their complete loss of nebulin and their dramatic phenotype that includes early mortality $21,22,27,35$.

Here we created a mouse model that recapitulates the typical nebulin-based NM patient with compound-heterozygous mutations. The model carries a p.Ser6366Ile missense mutation in one actin-binding sequence in super repeat 18 (super-repeat 22 in the mouse) and a deletion of Neb exon 55 . The missense mutation is a founder mutation with world-wide occurrence; in homozygous form it results in Finnish distal myopathy ${ }^{36}$, but it has also been identified in combination with other $N E B$ mutations resulting in compound-heterozygous $\mathrm{NM}^{36-38}$. In vitro studies with nebulin super-repeats harboring this p.Ser6366Ile mutation showed increased affinity for actin ${ }^{39}$. Deletion of NEB exon 55 was discovered as a founder mutation in Ashkenazi Jews, and has since been found to have global occurrence ${ }^{40,41}$. It results in intermediate or severe NM with diminished nebulin protein levels and muscle weakness ${ }^{35,42}$. By crossing the Ser6366Ile mouse model with our mouse model with $\mathrm{Neb}$ exon 55 deleted $^{35}$, a mouse was generated with compound-heterozygous $\mathrm{Neb}$ mutations. Functional, structural, and biochemical studies revealed altered thin filament structure, increased myofilament lattice spacing, a reduced myofibrillar fractional area, and reduced force production. This Compound-Het model will be useful for testing experimental therapies for typical NM.

\section{Results}

Creation and basic characterization of Compound-Het model. A mouse model was created with a c.19097G >T mutation in the NEB gene that results in a serine to isoleucine substitution at the position corresponding to human Ser6366 in nebulin superrepeat 18 (or 22 in the mouse: Fig. 1a, and Supplementary Fig. 1), the $\mathrm{Neb}^{56366 \mathrm{I}}$ model. Mice from this line produce heterozygous, homozygous and wildtype (WT) mice according to Mendelian genetic ratios (Fig. $1 \mathrm{~b}$ top). We bred heterozygous mice to heterozygous $\mathrm{Neb}^{\triangle \mathrm{Exon} 55}$ mice, which produced the expected genotypes according to Mendelian genetic ratios (Fig. 1b bottom). $\mathrm{Neb}^{\mathrm{S6366I} / \Delta \text { Exon55 }}$ and $\mathrm{Neb}^{\text {S6366I/S6366I }}$ mice are referred to as Compound-Het and $\mathrm{Neb}^{\text {S6366I }}$ Hom mice, respectively. Most studies used 4 months old mice, except when indicated otherwise. Compound-Het and $\mathrm{Neb}^{\text {S6366I }}$ Hom animals have no outwardly visible phenotype with no unusual deaths during the timespan at which they were studied (oldest mice in colony are 12 months old). Following mice over time revealed decreased body weights in the Compound-Het mice (Fig. 1c). Tibia lengths were slightly reduced ( 2\%) in both Compound-Het and Neb ${ }^{\text {S6366I }}$ Hom mice (Fig. 1d). Grip strength was decreased in Compound-Het mice, by $\sim 20 \%$ compared to WT mice (Fig. 1e). Neb ${ }^{\text {S6366I }}$ Hom mice had a $\sim 10 \%$ reduction at 10 mo (Fig. 1e). To study in situ muscle function, the force-frequency relation of the gastrocnemius muscle complex was measured with a hindlimb foot-plate system. In 3 mo old male mice of both genotypes forces were lower; the force reduction at the plateau of the force-frequency curve was $35 \%$ in Compound-Het mice and $7 \%$ in $\mathrm{Neb}^{\text {S6366I }}$ Hom mice (Fig. 1f, left). In 10 mo old mice a similar force reduction occurred in Compound-Het mice (37\%) and a significantly larger reduction in $\mathrm{Neb}^{\mathrm{S} 6366 \mathrm{I}}$ Hom mice (18\%) with identical results in female mice (Fig. 1f, middle and right). Normalized forcefrequency curves in both the Compound-Het and $\mathrm{Neb}^{\mathrm{S} 6366 \mathrm{I}} \mathrm{Hom}$ mice were left-shifted, relative to WT (Fig. 1g). The stimulation frequency required for generating half-maximal force was decreased in Compound-Het mice (Fig. 1g, inset).

Skeletal muscle atrophy/hypotrophy is a common finding in $\mathrm{NM}^{43,44}$, and muscle weights of the Compound-Het and $\mathrm{Neb}^{\text {S6366I }}$ Hom were compared with WT littermates. Muscle weights in Compound-Het mice were mostly decreased, a weight of a few muscle types was unchanged or increased (Fig. 2a). In $\mathrm{Neb}^{\mathrm{S} 6366 \mathrm{I}}$ Hom mice only two of the examined muscle types were changed: the gastrocnemius and plantaris had decreased weights (Fig. 2b). The decrease in plantaris weight was the same in the two genotypes (10\%) but the gastrocnemius muscle weight loss was more pronounced in Compound-Het $(20 \%)$ than $\mathrm{Neb}^{\mathrm{S} 6366 \mathrm{I}}$ Hom mice (9\%).

In summary, a Compound-Het mouse model $\left(\mathrm{Neb}^{\mathrm{S} 6366 \mathrm{I} / \Delta \text { Exon55)}}\right.$ was created with reduced body weight, an in-situ force deficit and altered muscle trophicity.

Compound-Het mice produce only $\mathrm{Neb}^{\mathrm{S6366I}}$ mutant protein. To determine which type of mutant nebulin is expressed in the Compound-Het muscles (i.e., gene product of $\mathrm{Neb}^{\mathrm{S} 6366 \mathrm{I}_{-}}$

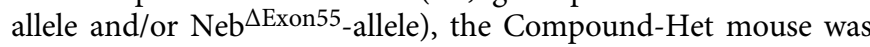
crossed to a mouse with a smaller nebulin protein achieved by genetically removing three super-repeats, i.e., $\mathrm{Neb}^{\Delta \mathrm{S} 9-11}$ (Kiss B. et al. in preparation). The smaller nebulin has a faster mobility during gel electrophoresis (deleting super-repeats 9-11 eliminates 729 amino acids) and this made it possible to identify the nebulin isoform in mice that contain the $\mathrm{Neb}^{\Delta \mathrm{S} 9-11}$ allele and either the $\mathrm{Neb}^{\mathrm{S} 6366 \mathrm{I}}$-allele or the $\mathrm{Neb}^{\Delta \text { Exon55-allele. By crossing }}$ the $\mathrm{Neb}^{\Delta \mathrm{S} 9-11}$ mouse with the $\mathrm{Neb}^{\text {S6366I }}$ mouse, a mouse is obtained that expresses a high mobility nebulin protein (produced by the $\mathrm{Neb}^{\Delta \mathrm{S9} 911}$-allele) and, if the $\mathrm{Neb}^{\mathrm{S6366I}}$-allele produces protein, a mutant protein with mobility similar to that of wildtype nebulin (the amino acid substitution in the $\mathrm{Neb}^{\mathrm{S} 6366 \mathrm{I}}$ changes the molecular mass by 26 Dalton only). Results reveal that the Neb ${ }^{\mathrm{S} 6366 \mathrm{I}}$-allele indeed produces nebulin protein (Fig. 3a). By crossing the Neb $\mathrm{NS}^{\Delta-11}$-mouse with the Neb ${ }^{\Delta \text { Exon55. }}$ mouse a compound-heterozygous mouse was created with a high mobility nebulin protein (again produced by the $\mathrm{Neb}^{\left.\Delta \mathrm{S} 9-11^{-a l l e l e}\right)}$ and, if the $\mathrm{Neb}^{\Delta \text { Exon55-allele produces nebulin, a mutant }}$ protein with mobility similar to that of wildtype nebulin (exon 55 encodes 35 amino acid residues with a combined $4 \mathrm{kDa}$ mass, a too-small size difference to cause a mobility shift on gels). Protein gels showed that none of the studied muscle types produce full-length nebulin at detectable levels, i.e., the Neb ${ }^{\Delta \text { Exon } 55}$ allele does not produce protein (Fig. 3a). Thus, it is likely that the nebulin protein produced by the Compound-Het mouse $\left(\mathrm{Neb}^{\mathrm{S} 6366 \mathrm{I} / \Delta \text { Exon55)}}\right)$ is $\mathrm{Neb}^{\mathrm{S6366I}}$-nebulin. 
a

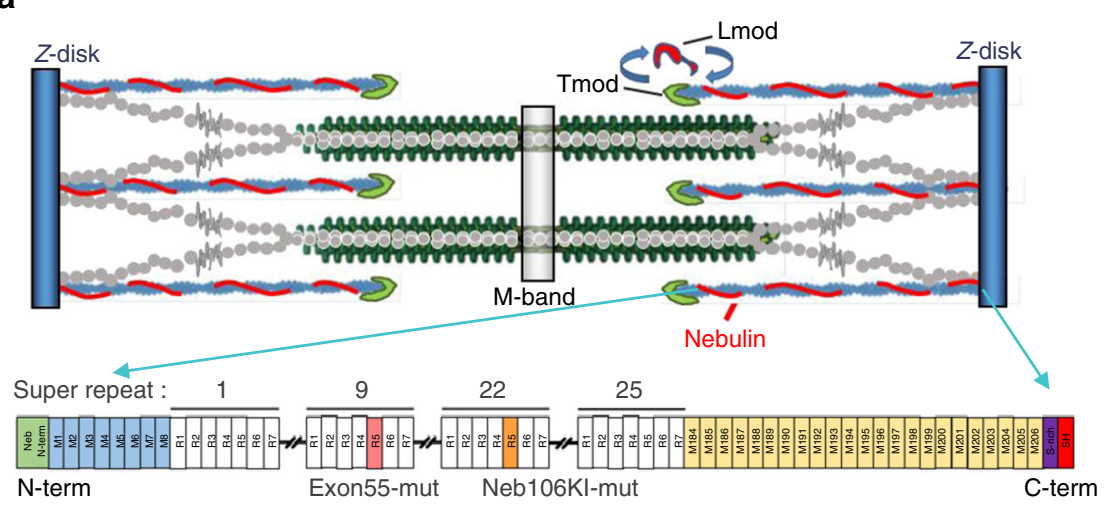

b
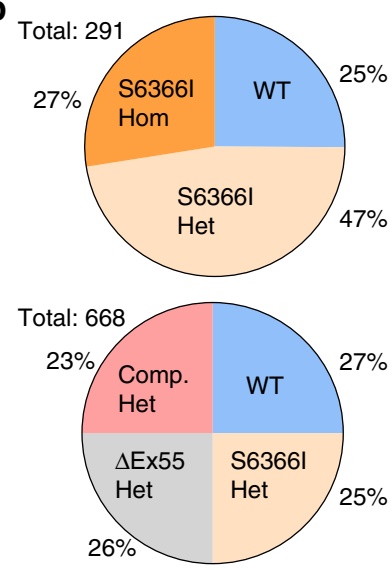

C

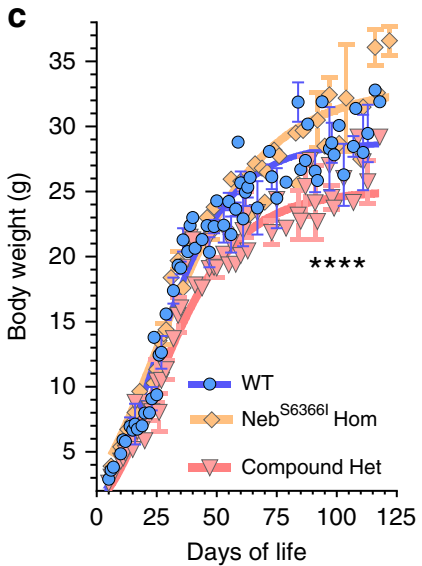

d

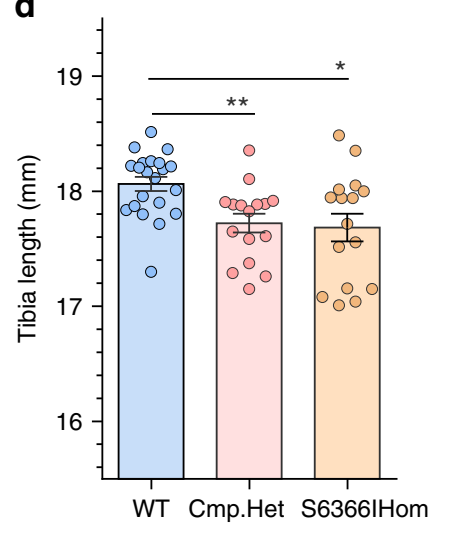

e

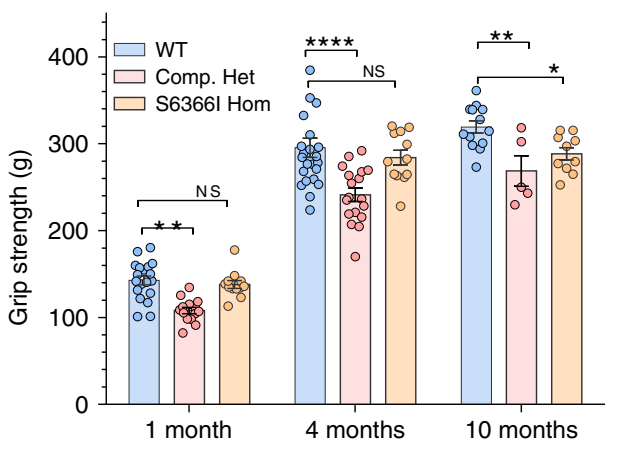

f
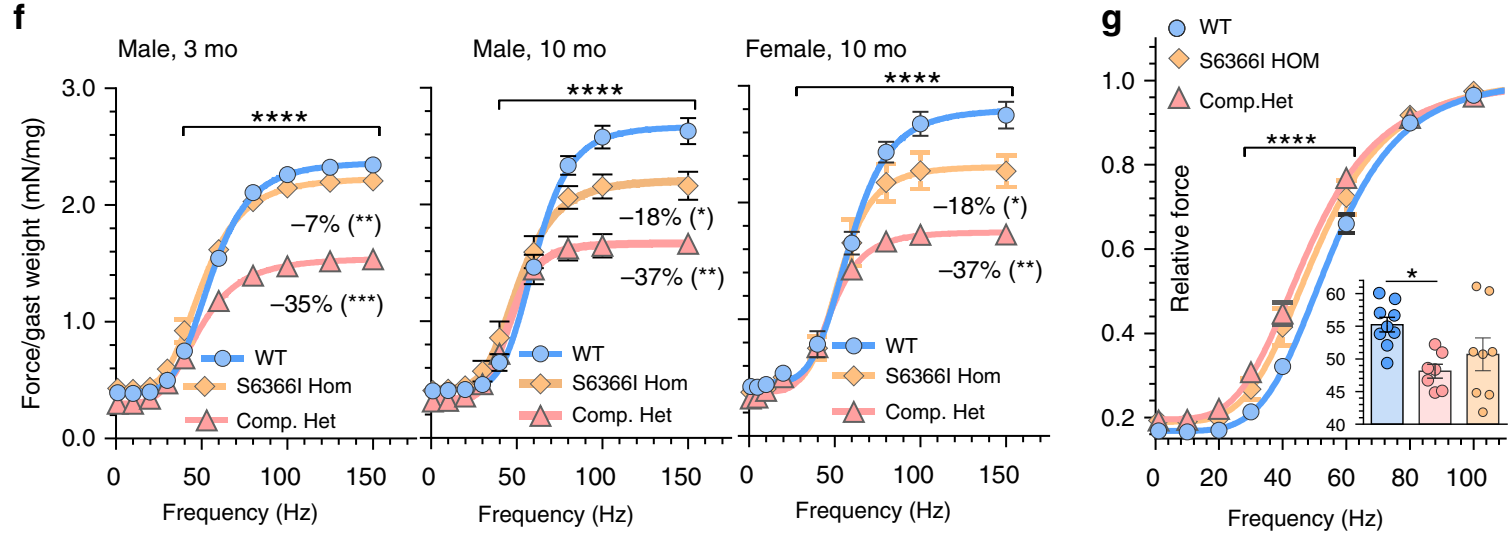

As other models of nebulin-based NM have decreased nebulin protein levels ${ }^{21,22,27,35}$, we investigated the nebulin content in diaphragm and lower limb muscles in Compound-Het and $\mathrm{Neb}^{\mathrm{S} 6366 \mathrm{I}}$ Hom animals. Nebulin protein levels were maintained at WT levels in all examined muscles from both genotypes (Fig. 3b, c). Thus, the Compound-Het mouse model

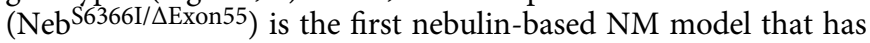
normal nebulin protein levels.

Changes in myosin heavy chain distribution. A common finding in patients with nebulin-based NM and mouse models are changes in the proportion of myosin heavy chain (MHC) isoforms ${ }^{27,29,44}$. The Compound-Het mice $(4 \mathrm{mo})$ followed this general trend and with the exception of the tibialis cranialis all examined muscles had changes in MHC expression (Fig. 4a). Soleus muscles had the most prominent change in type I myosin,

increasing from $\sim 40 \%$ to $\sim 70 \%$. In most other muscles, type IIB myosin proportion decreased while IIA/X increased (Type IIA and IIX cannot be separated in our experiments and are quantified as a single band). Neb ${ }^{\text {S6366I }}$ Hom animals, interestingly, displayed no changes in MHC proportion in any examined muscle type (Supplementary Fig. 2a). We also studied MHC isoform distribution in 2 mo and 10 mo old Compound-Het mice and focused on the EDL, soleus and TC muscles. Significant changes in the EDL and soleus muscle already existed in the 2 mo old mice and they persisted in the 10 mo old mice; a small shift in 2 mo and 10 mo old TC muscle was also found (Supplementary Fig. 2b). The same muscle types were also studied in 10 mo old S6366I mice which revealed in EDL and soleus muscle small but significant shifts toward MHC isoforms found in oxidative fibers (Supplementary Fig. 2c).

To gain additional insights into the fiber-type changes, crosssections of EDL and soleus muscles were stained with antibodies 
Fig. 1 Basic characterization of the mouse strains. a Top: Nebulin localizes to the thin filament of the skeletal muscle sarcomere. Bottom: The structure of mouse nebulin with the $\mathrm{S6366I}$ site indicated (in super-repeat 22, orange) and the $\Delta$ Exon55 site (in super-repeat 9, pink). A mouse was created with

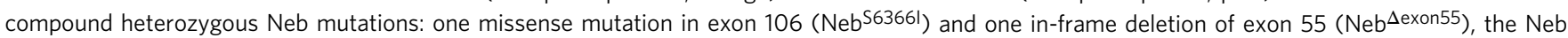

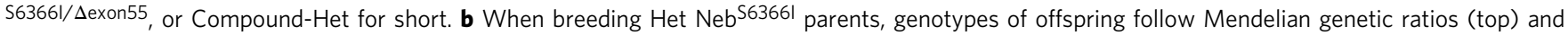
when breeding Het $\mathrm{Neb}^{\mathrm{S} 63661}$ with Het $\mathrm{Neb}^{\Delta \mathrm{exon} 55}$ parents (bottom), offspring also closely follows Mendelian genetics. c Body weight vs. days-of-life. Gompers non-linear least squares fit with as null hypothesis that one curve fits all data sets. Test (Sum-of-squares F-test) reveals that the null hypothesis is rejected ( $p \leq 0.0001 ; F(D F n, D F d): 63(3,341)$ ). (Results of male mice are shown with similar results found for female mice (not shown).) $\mathbf{d}$ Tibia length is reduced in Compound-Het and $\mathrm{Neb}^{\mathrm{S} 63661}$ homozygous 4 months old male mice. An ordinary one-way ANOVA without matching or paring was performed with a posthoc multiple testing comparison with multiple testing correction (Sidak). ${ }^{\star} p=0.02 ;{ }^{\star \star} p=0.008$. e Grip strength in Compound-Het and $\mathrm{Neb}^{563661}$ mice, 1, 4 and 10 mo of age. A two-way ANOVA (no matching) reveals a significant effect of age and genotype on grip strength but without interaction. A posthoc multiple testing comparison with multiple testing correction (Dunnett) reveals a significant grip strength reduction at all 3 ages in Compound-Het mice and a significant reduction in 10 mo old Neb ${ }^{563661}$ mice. ${ }^{\star \star} p<0.01$; ${ }^{\star \star \star *} p<0.0001$. $\mathbf{f}$ In 3 mo old male mice (left) Compound-Het mice have a $35 \%$ decreased maximal lower limb force and $\mathrm{Neb}^{563661} \mathrm{Hom}$ have a $7 \%$ reduction. In 10 mo old male mice (middle) the reduction is $37 \%$ and $18 \%$ and in 10 mo old female mice (right) the reduction is $37 \%$ and $18 \%$. Non-linear least squares fit to Hill curve with as null hypothesis that one curve fits all

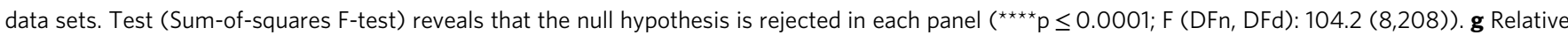
force-frequency relation (same data as in $\mathrm{f}$, left) shows a left shift for both the Compound-Het and $\mathrm{Neb}^{563661}$ Hom mice with increased relative force at the shown frequencies of 40 and $60 \mathrm{~Hz}$ (One-way ANOVA without matching or paring performed with a posthoc multiple testing comparison with multiple testing correction (Sidak). ${ }^{\star \star \star \star} p<0.0001$. Inset: decreased frequency for $1 / 2$ maximal force in Compound-Het mice. Ordinary one-way ANOVA without matching or paring. A posthoc multiple testing comparison with multiple testing correction (Sidak). ${ }^{*} p=0.02$. Number of mice in $\mathbf{b} 291$ (top) and 668 (bottom); c, d: WT: 21, Compound-Het: 18, and NebS6366I Hom: 16; e 1 mo 19, 14, 12; 4 mo: 22, 18, 12; 10 mo 14, 6, 10. f left and g: WT: 9, Compound-Het:

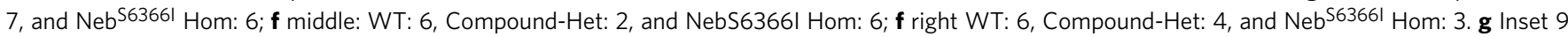
WT mice, 6 Compound-Het mice and $7 \mathrm{Neb}{ }^{\mathrm{S} 63661} \mathrm{Hom}$ mice. Bar values are mean \pm SEM. Source data are provided as a Source Data file.
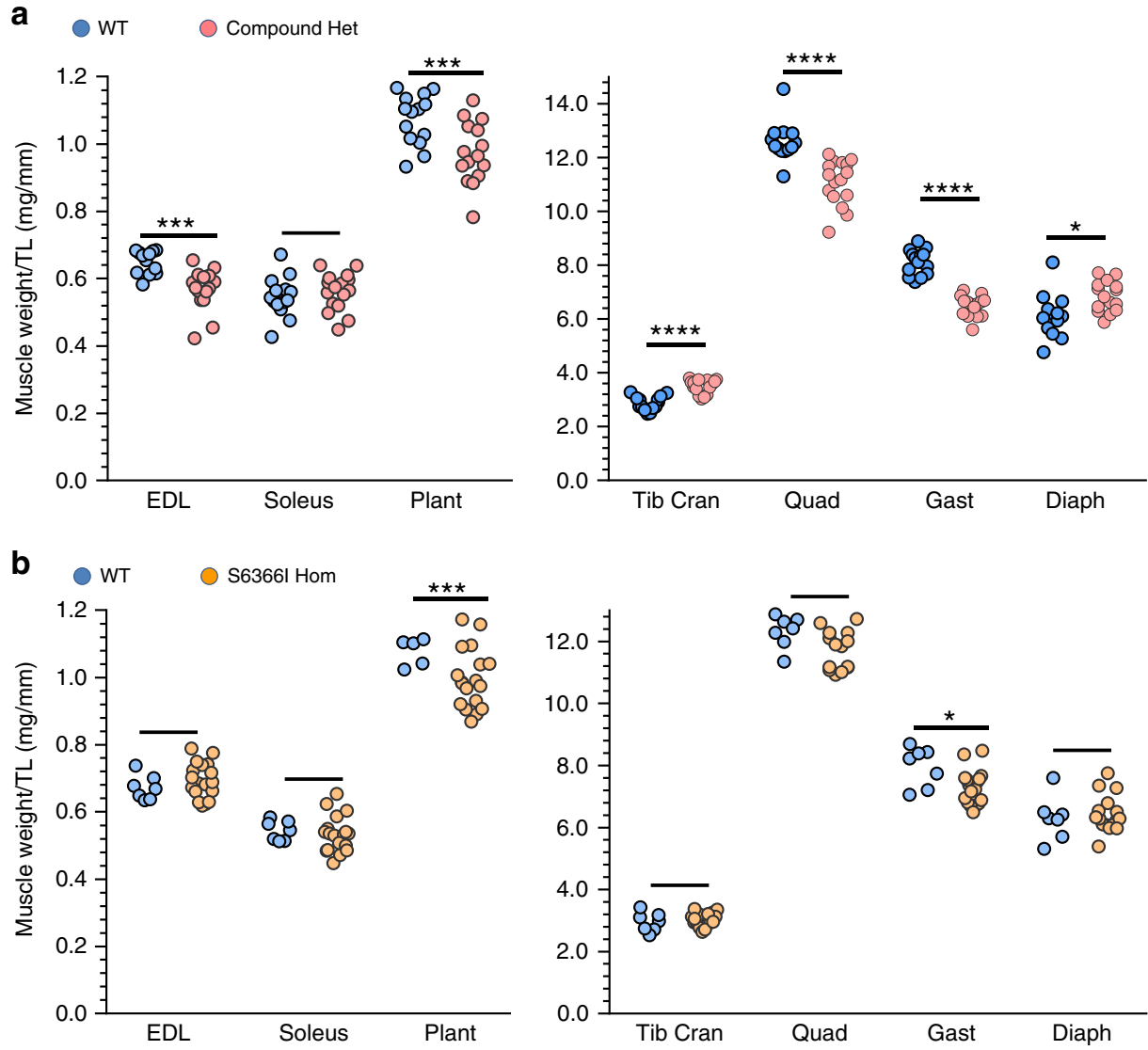

Fig. 2 Assessing muscle weights. a Compound-Het mice have decreased tissue weight in EDL, plantaris, quadriceps and gastrocnemius muscles while muscle weight of tibialis cranialis and diaphragm is increased. b Neb ${ }^{563661}$ Hom mice have decreased muscle weight in plantaris and gastrocnemius. Number of mice in a: WT: 14, Compound Het: 16. Number of mice in b: WT: 7, and Neb ${ }^{563661}$ Hom: 18. (Male mice (4 mo); similar findings in female mice, not shown; 2-Way ANOVA with multiple comparison (multiple comparison correction (Sidak)). ${ }^{\star} p<0.05 ;{ }^{\star \star \star} p<0.001 ;{ }^{* \star \star \star} p<0.0001$ ). Source data are provided as a Source Data file. 

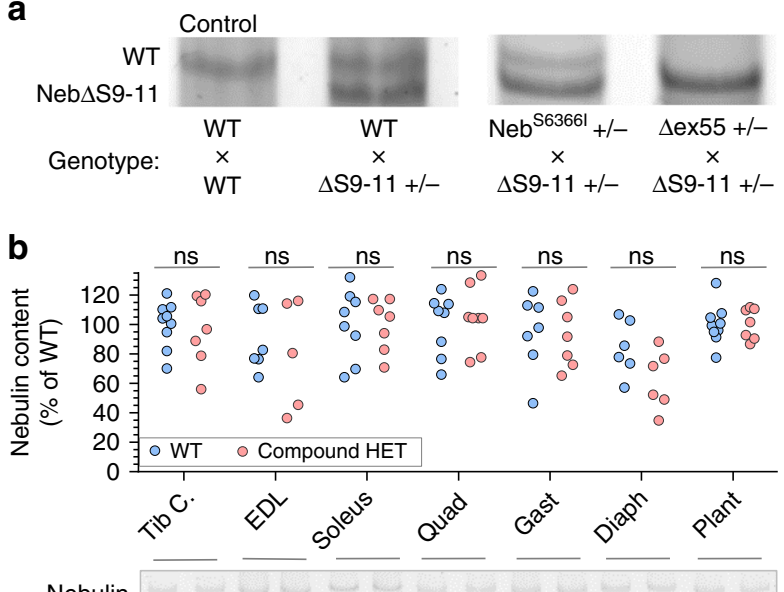

Nebulin

$\mathrm{MHC}$

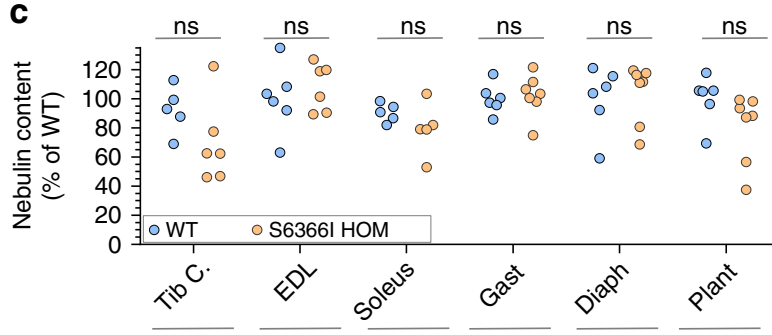

Nebulin

$\mathrm{MHC}$

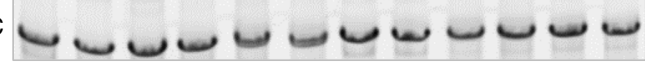

Fig. 3 Nebulin protein expression. a Nebulin isoform expression was assessed in muscles from mice with one $\mathrm{Neb}^{\Delta \mathrm{S} 9-11}$-allele (this expresses a nebulin lacking super-repeats 9-11, generating a smaller than wild type nebulin) and the other allele either WT, Neb ${ }^{563661}$ or $\mathrm{Neb}^{\Delta \mathrm{ex} 55}$. The latter two alleles are expected to produce a nebulin protein of wildtype size (assuming they express protein, see text for details). Top: protein gels with nebulin protein bands; bottom: genotype of mouse from which muscle was obtained (gastrocnemius muscle). The left lane is from a WT mouse revealing WT nebulin and the second lane is from a heterozygous $\mathrm{Neb}^{\Delta S 9-11}$ mouse that reveals WT nebulin (top band) and a bottom nebulin band produced by the $\mathrm{Neb}^{\Delta \mathrm{S} 9-11}$ allele. Lanes 3 and 4 show that the $\mathrm{Neb}^{\mathrm{S63661}}$ allele produces full-length nebulin but that the $\mathrm{Neb}{ }^{\Delta \mathrm{ex} 55}$ does not. Identical results (not shown) were obtained in other examined muscle types (EDL, soleus Tibialis C. gastrocnemius and diaphragm), two mice per genotype with 6 muscle types studied per mouse. See text for details. b, c Expression levels of nebulin relative to myosin heavy chain $(\mathrm{MHC})$ in a range of muscle types. Analyzed results (top) and gel examples (bottom) of a Compound-

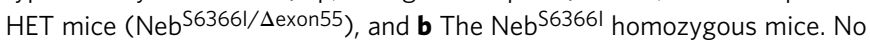
differences in nebulin protein levels were found in any of the studied muscle types. 2-Way ANOVA with multiple comparison (multiple comparison correction (Sidak)). ns not significant. Number of mice in $\mathbf{b}$ : WT: 9 , Compound-Het: 8. Number of mice in c: WT: 6, and S6366I Hom: 7. Source data are provided as a Source Data file.

against the different myosin isoforms to determine fiber size and number (Fig. 4b, c left panels show examples). Because minimal changes were present in MHC expression in $\mathrm{Neb}^{\mathrm{S} 6366 \mathrm{I}} \mathrm{Hom}$ animals (see above), we focused this part of the work on only the Compound-Het mice. EDL from Compound-Het mice had a small reduction in the minFeret of type IIX fibers while the minFeret of the relatively few type I fibers doubled (Fig. $4 \mathrm{~b}$ middle). The size of type IIB fibers was unchanged but the number of type IIB fibers was decreased by $20 \%$ in CompoundHet EDL muscles (Fig. $4 \mathrm{~b}$ right). It is likely that the reduction in the number of type IIB fibers underlies the reduced EDL muscle mass. Soleus muscle from Compound-Het mice had a 50\% decrease in type IIX minFeret and for IIA fibers minFeret trended down (Fig. 4c middle). The number of type I fibers was doubled while the number of type IIA fibers decreased by $\sim 40 \%$ (Fig. $4 \mathrm{c}$ right). The large increase in the number of type I fibers is likely to explain the preserved soleus muscle mass in CompoundHet mice.

Ultrastructural and histological effects of mutant nebulin. Electron microscopy revealed nemaline rod bodies in EDL from both Compound-Het and $\mathrm{Neb}^{\mathrm{S} 6366 \mathrm{I}}$ Hom mice that typically originated from the Z-disk (Fig. 5, yellow arrows). Interestingly, in soleus muscle rod bodies were only present in Compound-Het mice (Fig. 5a) and they are smaller and take up less fractional area than in EDL muscle (Fig. 5b, c). Wavy and misaligned Z-disks and areas with damaged Z-disks were also present in muscles from Compound-Het and $\mathrm{Neb}^{\mathrm{S} 6366 \mathrm{I}}$ Hom mice (Fig. 5a). The presence of rod bodies has been examined in biopsies of Finnish distal myopathy patients that are homozygous for the Neb ${ }^{\mathrm{S} 63661}$ mutation $^{36}$. (This mutation was initially designated p.Ser4665$\left.\mathrm{Ile}^{36}\right)$. Only few and small nemaline rod bodies could be detected ${ }^{36}$. This earlier study on distal myopathy patients used histology with modified Gomori trichrome staining and we therefore also investigated rod bodies in gastrocnemius muscle of the mouse models, using the same method as used on patients. Gomori trichrome stained sections showed rod bodies in Compound-Het mice at both ages (Supplementary Fig. 3b, e). Furthermore, we detected small rod bodies in S6366I Hom mice at four and ten months of age (Supplementary Fig. 3c, f). These histology results in S6366I Hom mice are in overall agreement with those of the Finnish S6366I patients ${ }^{36}$. It is also noteworthy that core-like structures were observed in ten months old Compound-Het mice (Supplementary Fig. 3e). Cores have also been observed in myopathy patients with nebulin mutations ${ }^{3,45}$.

Shorter thin filaments are frequently found in nebulin-based $\mathrm{NM}^{46,47}$, and thin filament lengths were therefore measured on electron micrographs in Compound-Het and $\mathrm{Neb}^{56366 \mathrm{I}} \mathrm{Hom}$ muscles. EDL muscles were studied because their $\mathrm{H}$-zone can relatively easily be discerned, and thin filament lengths can be measured as the distance from the edge of the $\mathrm{H}$-zone to the middle of the Z-disk (Supplementary Fig. 4a). Unlike what has been found in previous nebulin-based NM-studies, no decrease in thin filament lengths were detected. Instead a slight but significant increase in thin filament length was detected in both Compound-Het and $\mathrm{Neb}^{\mathrm{S} 6366 \mathrm{I}}$ Hom muscles (Fig. 5d and Supplementary Fig. 4a-c). To validate this result, superresolution optical microscopy was used to measure thin filament length in EDL muscle by labeling the thin-filament capping protein $T \bmod 1{ }^{48}$. This also revealed longer thin filaments in Compound-Het mice, compared to WT (Supplementary Fig. 4d). Thick filament lengths, measured by using the Ti102 antibody that labels the edge of the thick filament ${ }^{49}$, revealed no differences (Fig. S4e).

Influence of mutant nebulin on whole muscle function. Muscle function of EDL and soleus was studied by electrically stimulating isolated intact muscles at a range of frequencies and measuring at the optimal length $\left(\mathrm{L}_{0}\right)$, isometric force. The maximal specific force produced by EDL muscle was reduced by $~ 22 \%$ in Compound-Het and $\sim 17 \%$ in $\mathrm{Neb}^{563661}$ Hom muscles (Fig. 6a inset). Normalizing the measured force at each frequency by the 


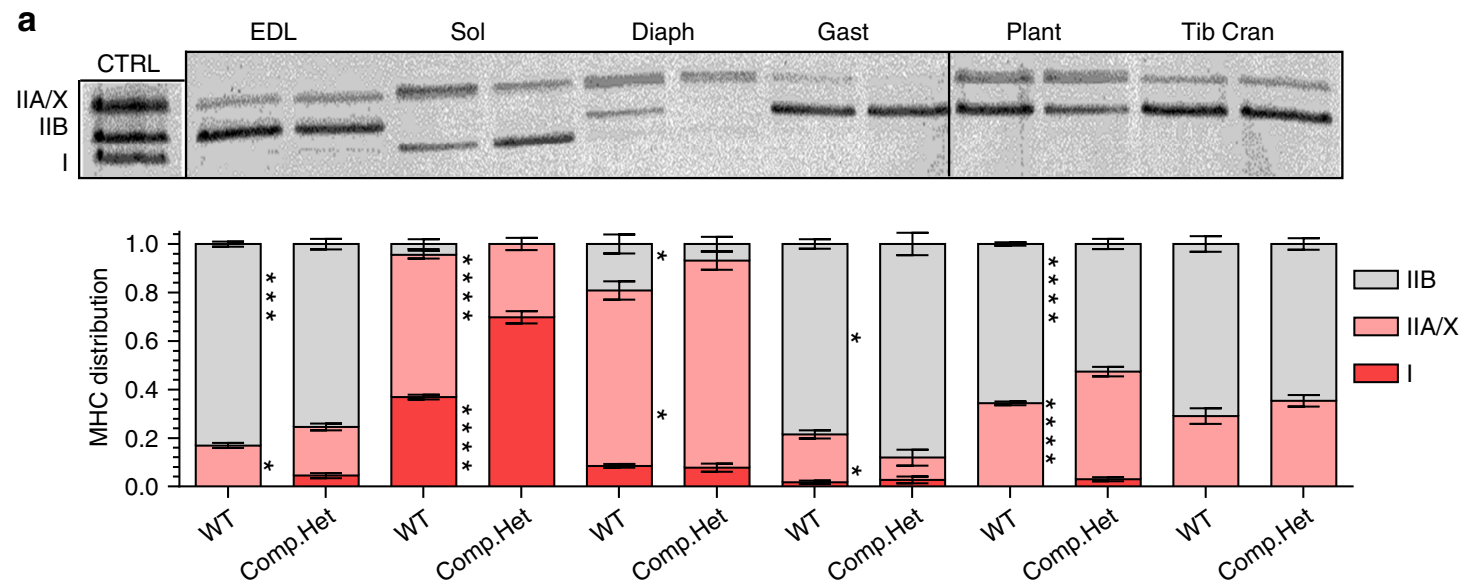

b
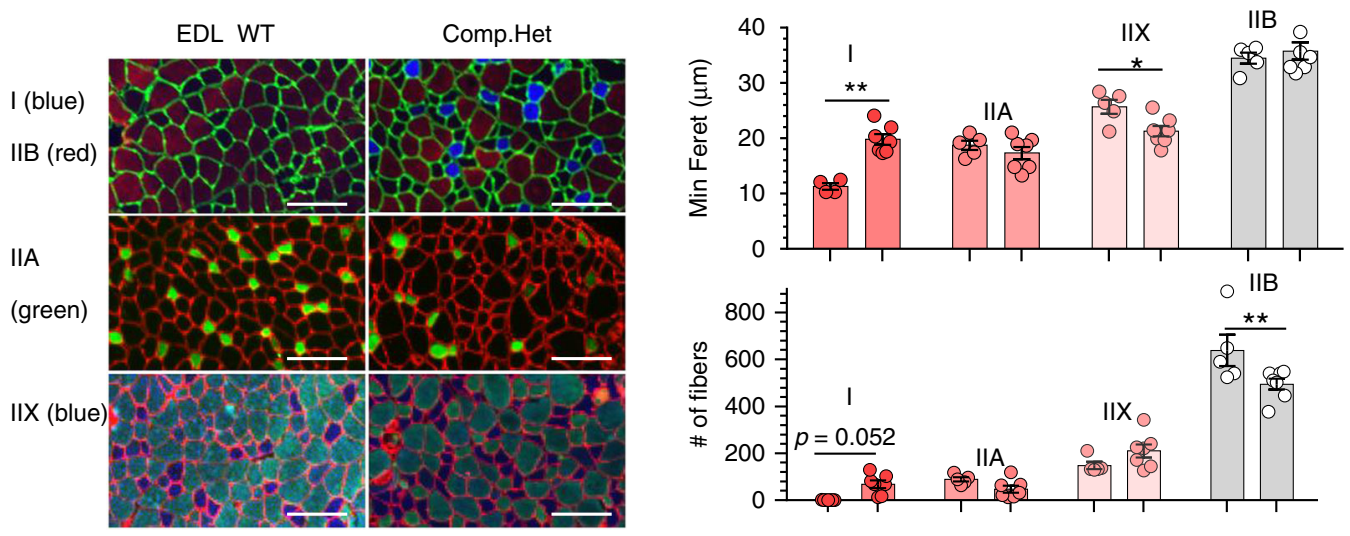

C
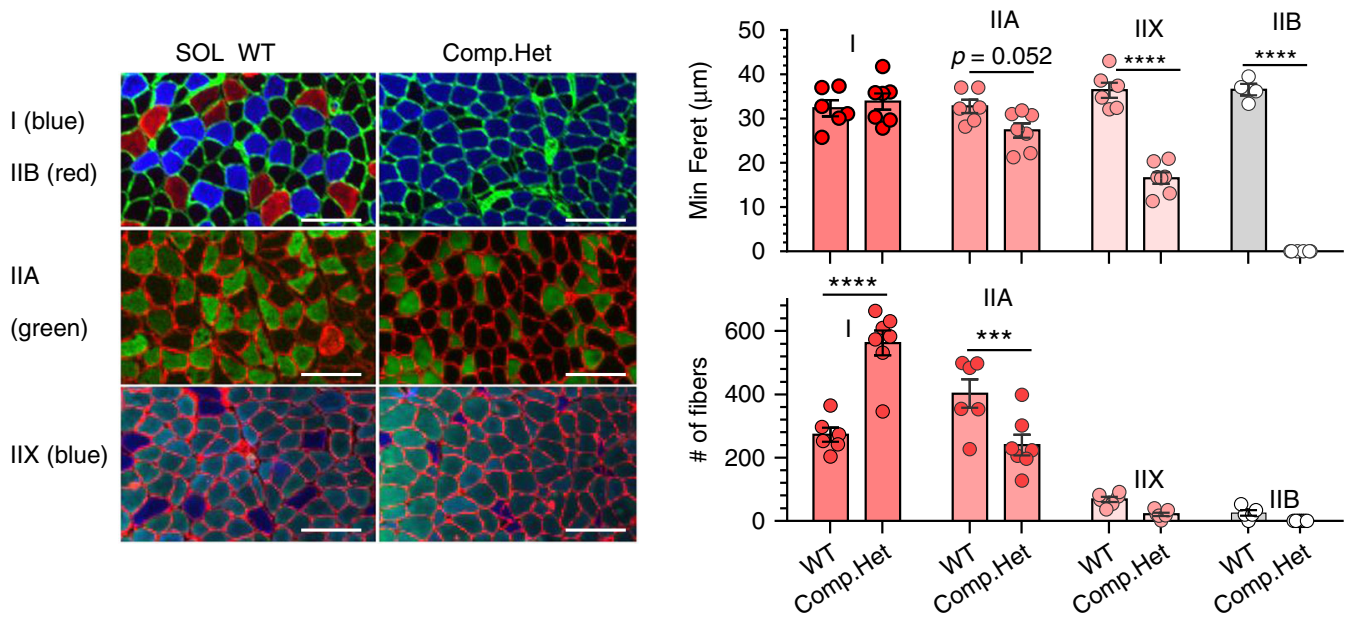

Fig. 4 Myosin heavy chain (MHC) isoform distribution and CSA analysis. a MHC expression in Compound-Het animals display significant changes in MHC distribution. A shift away from type IIB and toward slower myosin isoforms (either type IIA/X or type I) is observed in most muscle types (an exception is the tibialis m.). Top representative MHC gel images; bottom analyzed results. The control (CTRL) is a pooled homogenate of TC and Soleus muscle, revealing the 3 isoforms that can be discerned: type I, IIA/X and IIB (from bottom to top). $\mathbf{b}$ Example cross-sections stained with antibodies that are MHC-type specific (left), minFeret diameter (right, top) and number of fibers (right, bottom) in EDL muscle. MinFeret is increased in type I fibers and reduced in IIX. The number of type I fibers number is increased (only 3 type I fibers were found in WT mice) and the number of type IIB fibers is reduced. Scale bar: $100 \mu \mathrm{m}$. c Example cross-sections stained with antibodies that are MHC-type specific (left), minFeret diameter (right, top) and number of fibers (right, bottom) in soleus muscle. There is a reduction in type IIX and IIB minFeret and an increase in the number of type I and a reduced number of type IIA fibers. Scale bar: $100 \mu \mathrm{m}$. a-c: Two-way ANOVA (no matching) with a posthoc multiple testing comparison with multiple testing correction (Sidak). ${ }^{\star} p<0.05,{ }^{\star \star} p<0.01 ;{ }^{* \star \star} p<0.001 ;{ }^{\star \star \star *} p<0.0001$. Number of mice in $\mathbf{a}:$ WT: 9, Compound-Het: 7. Number of mice in b and $\mathbf{c}$ : WT: 6, Compound-Het: 7. Bar values are mean \pm SEM. Source data are provided as a Source Data file. 
a
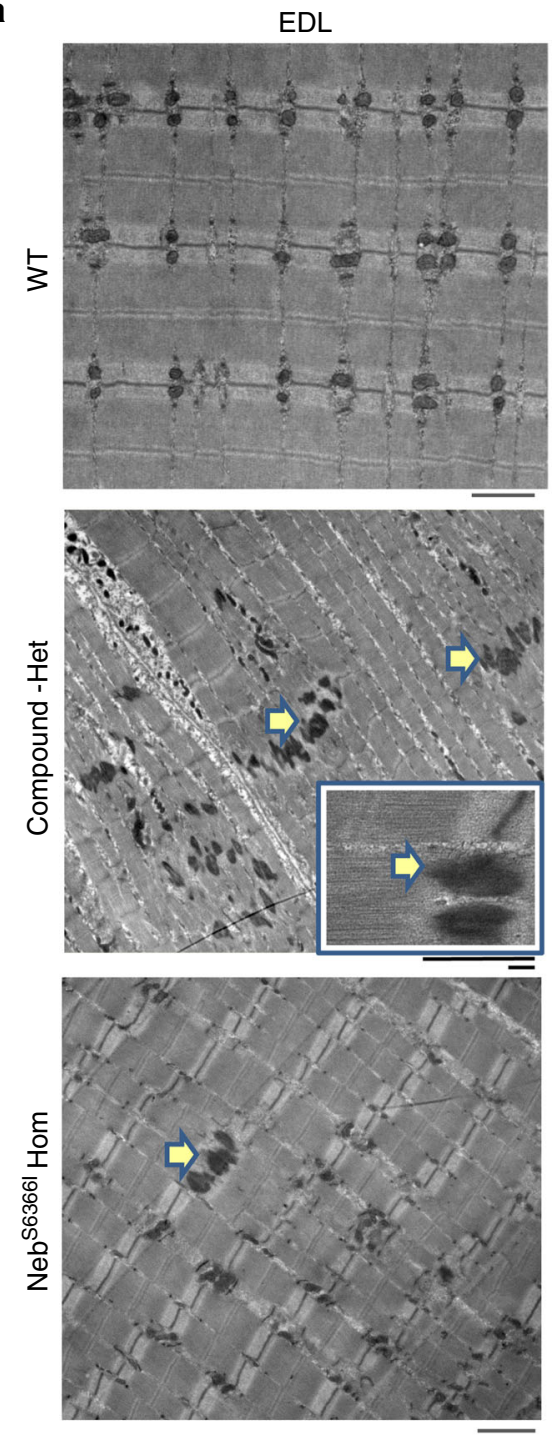

Soleus
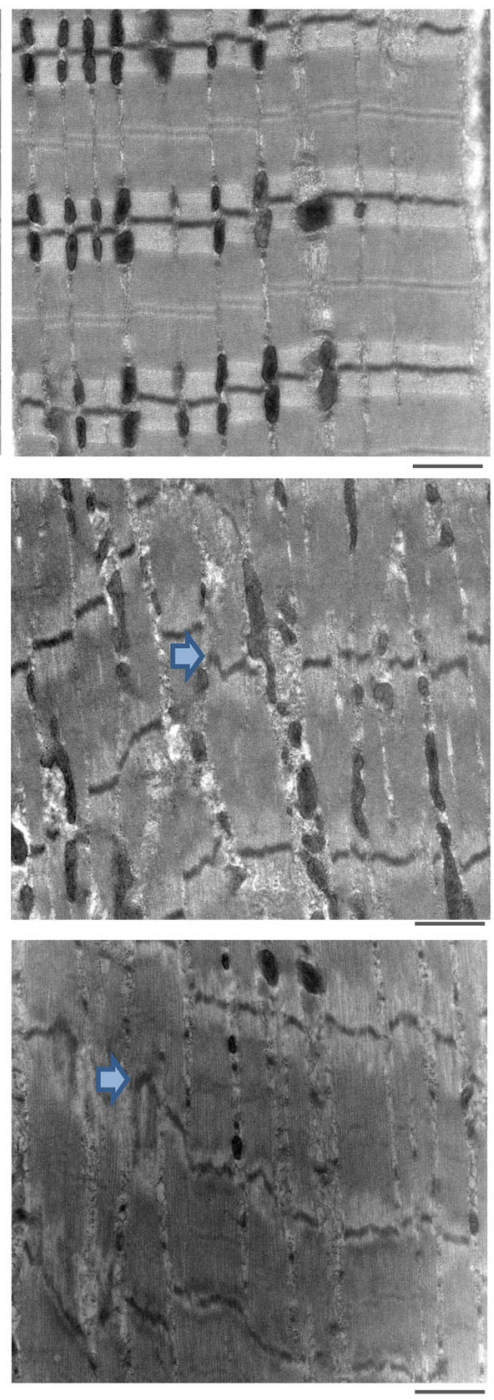

b

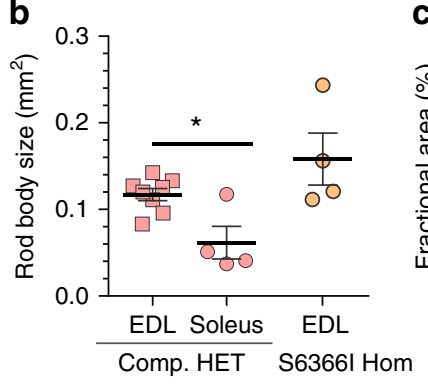

C

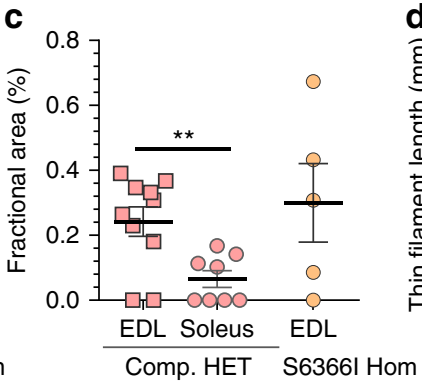

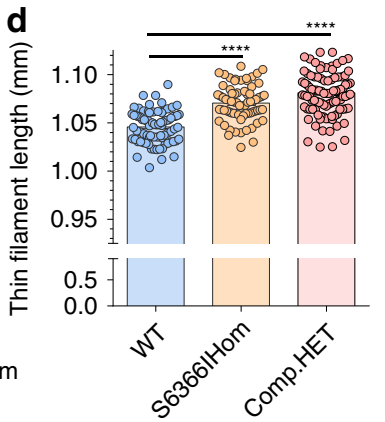

Fig. 5 Nemaline rod bodies, sarcomeric ultrastructure, and thin filament length. a Electron micrographs of intact EDL muscles (left) and soleus muscle (right) in WT (top), Compound-Het (middle) and $\mathrm{Neb}^{\mathrm{S63361}}$ How (bottom). Nemaline rod bodies (yellow arrows) are found in both EDL and soleus of Compound-Het and in only EDL of $\mathrm{Neb}^{\mathrm{S} 63661} \mathrm{HOM}$ mice. Inset in left middle panel shows enlarged rod bodies. In soleus muscle sarcomere disorganization was frequently seen in both Compound-HET and Neb ${ }^{563661} \mathrm{HOM}$ mice. Yellow arrows: rod bodies; blue arrows: altered/damaged Z-disks. Rod body size (b) and fractional area of rod bodies (c) in EDL and soleus. $\mathbf{b}{ }^{\star} p=0.03$. $\mathbf{c}{ }^{\star \star} p=0.0059$. Values are means \pm SEM. Four mice per genotype were used with $\sim 5$ fibers per muscle examined. The mean value for each mouse is plotted. $\mathbf{d}$ Thin filament lengths are slightly but significantly longer in Compound Het and $\mathrm{Neb}^{\mathrm{S} 63661} \mathrm{Hom}$ EDL muscle. Measurements made in SL range: 2.5-3.0 $\mu \mathrm{m}$. (See Supplementary Fig. 4 for images and additional data, including confirmations by super-resolution optical microscopy.) b-d Ordinary one-way ANOVA without matching or paring. A posthoc multiple testing comparison with multiple testing correction (Tukey) was performed. $\mathbf{d}^{\star \star \star \star} p<0.0001$. $n(W T)=81$ measurements from 18 fibers from 6 mice; $n($ Hom) $=71$ measurements from 18 fibers from 6 mice; $n($ Compound HET) $=103$ measurements from 18 fibers from 6 mice. Horizontal lines are mean \pm SEM. Source data are provided as a Source Data file. 


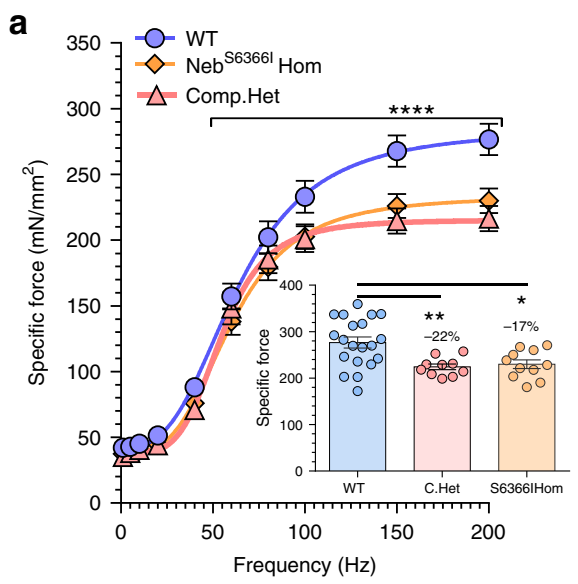

C
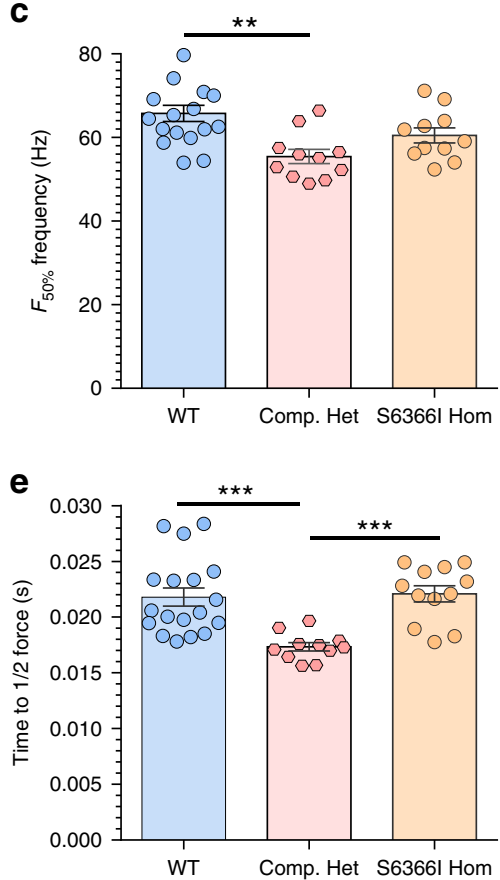

b

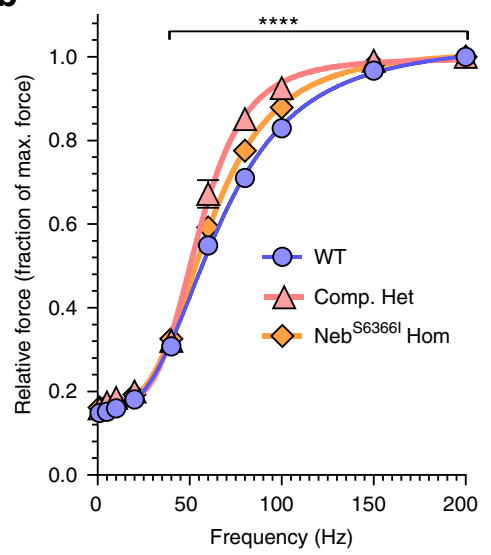

d

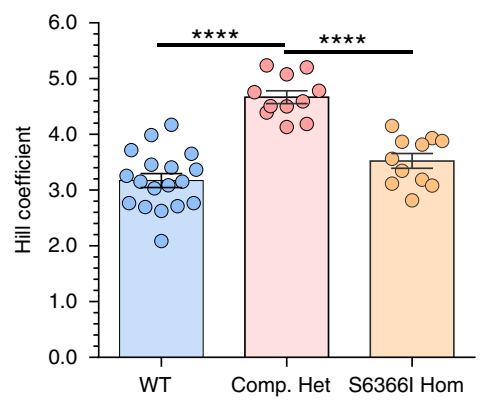

f

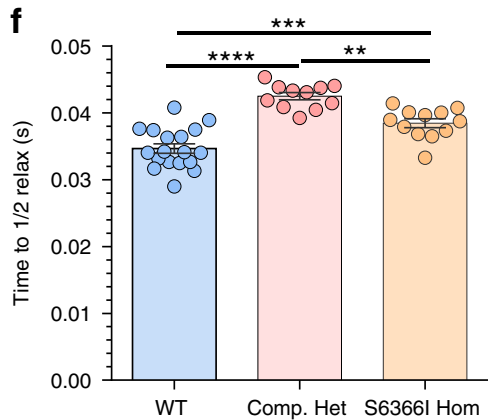

Fig. 6 Muscle mechanics. a Force-frequency relation of WT, Compound-Het and S6366I Hom EDL muscle. Inset shows maximal force (200 Hz stimulation rate). Force is reduced in Compound-Het and $\mathbf{5 6 3 6 6 1 ~ H o m ~ m i c e . ~ ( F o r c e ~ e x p r e s s e d ~ a s ~ s p e c i f i c ~ f o r c e : ~ f o r c e ~ p e r ~ u n i t ~ a r e a ~ o f ~ m u s c l e , ~} \mathrm{mN} / \mathrm{mm}{ }^{2}$ ). ${ }^{\star} p=0.013$, ${ }^{\star \star} p=0.007$. b Relative force-frequency shows steeper force rise in Compound-Het mice. c Frequency for $50 \%$ force $\left(F_{50 \%}\right)$ is reduced in Compound-Het mice. ${ }^{\star \star} p=0.0012$. d Hill coefficient is increased in Compound-Het mice. ${ }^{\star \star \star \star} p<0.0001$. e Time to $1 / 2$ maximal force of $200 \mathrm{~Hz}$ tetanus is reduced in Compound-Het mice. ${ }^{\star \star \star} p=0.0005$. f Time to $1 / 2$ relaxation of $200 \mathrm{~Hz}$ tetanus is increased in Compound-Het mice and S6366I Hom mice. ${ }^{* \star} p=0.002$, ${ }^{\star \star \star} p=0.0008$ and ${ }^{* \star \star \star} p<0.0001$. a-f: $n(\mathrm{WT})=20$ mice; $n$ (Compound-Het) $=11$ mice; $n($ S6366I Hom $)=11$ mice. Bar values are mean \pm SEM. a, b Nonlinear least squares fit to Hill curve with as null hypothesis that one curve fits all data sets. Test (Sum-of-squares F-test) reveals that the null hypothesis is rejected in each panel ( $p \leq 0.0001, \mathrm{~F}$ (DFn, DFd) $9.633(8,408)$ ). a inset and $\mathbf{c}-\mathbf{f}$ : ordinary one-way ANOVA without matching or paring. A posthoc multiple testing comparison with multiple testing correction (Tukey) was performed. Source data are provided as a Source Data file.

maximal force $(200 \mathrm{~Hz})$ revealed for the Compound-Het muscles a force increase at sub-maximal stimulations with a corresponding decrease in the frequency for half-maximal force, and increased cooperativity (Fig. 6b-d). EDL from Compound-Het mice took a shorter time to produce half-maximal force during tetanic stimulation (Fig. 6e) but longer time to half relaxation (Fig. 6f). Neb ${ }^{\text {S6366I }}$ Hom EDL muscles also relaxed more slowly, as indicated by the longer time for half relaxation, but not as long as for Compound-Het muscles (Fig. 6f). Soleus muscles had preserved specific force in Compound-Het and $\mathrm{Neb}^{\mathrm{S6366I}}$ Hom muscles (Supplementary Fig. 5a). The Compound-Het soleus muscle had a reduced frequency for half-maximal activation, and an increased Hill-coefficient and slowed relaxation time (Supplementary Fig. 5b-d, f). The $\mathrm{Neb}^{\mathrm{S} 6366 \mathrm{I}}$ Hom soleus muscle only had slowed relaxation times (Supplementary Fig. 5f). In summary, EDL muscle from both mouse models had impaired force production and slowed relaxation whereas the phenotype in soleus muscle was much milder. The mechanistic basis of these finding was studied next, using single fiber mechanics and X-ray diffraction on EDL muscles from Compound-Het mice, where the contractility phenotype was most severe.

Changes in single fiber force and calcium sensitivity. To establish whether the depressed contractility of intact muscle in Compound-Het mice has a myofilament-basis, single fiber studies were conducted. Membrane-permeabilized fibers from EDL muscles were used and force-pCa-curves $\left(\mathrm{pCa}=-\log \left(\left[\mathrm{Ca}^{2+}\right]\right)\right.$, 
a
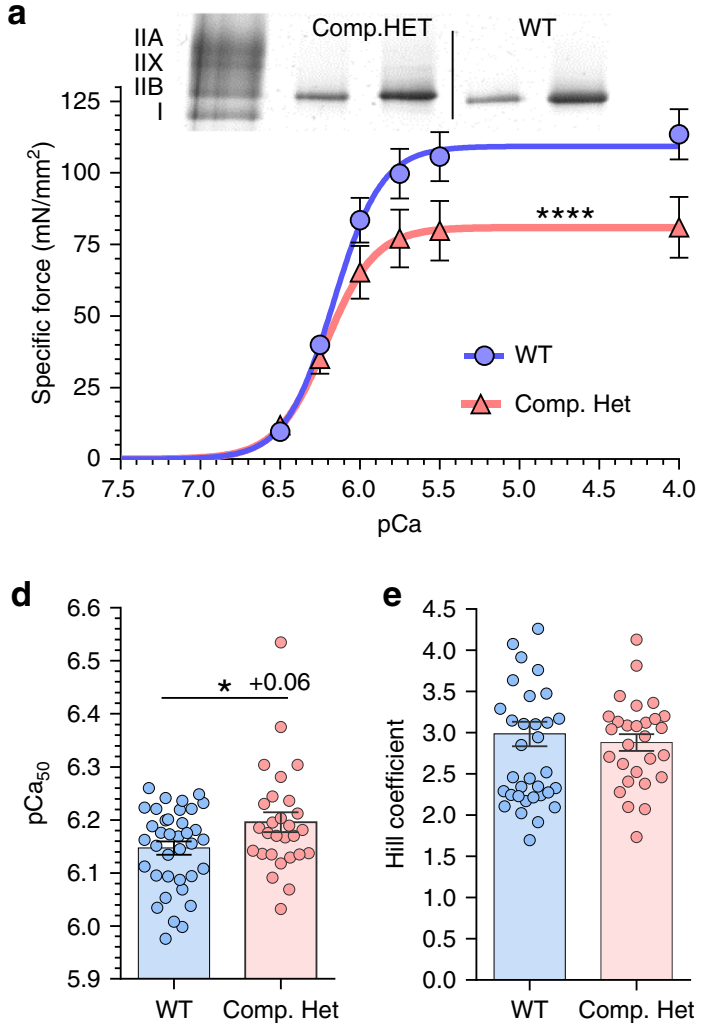

b
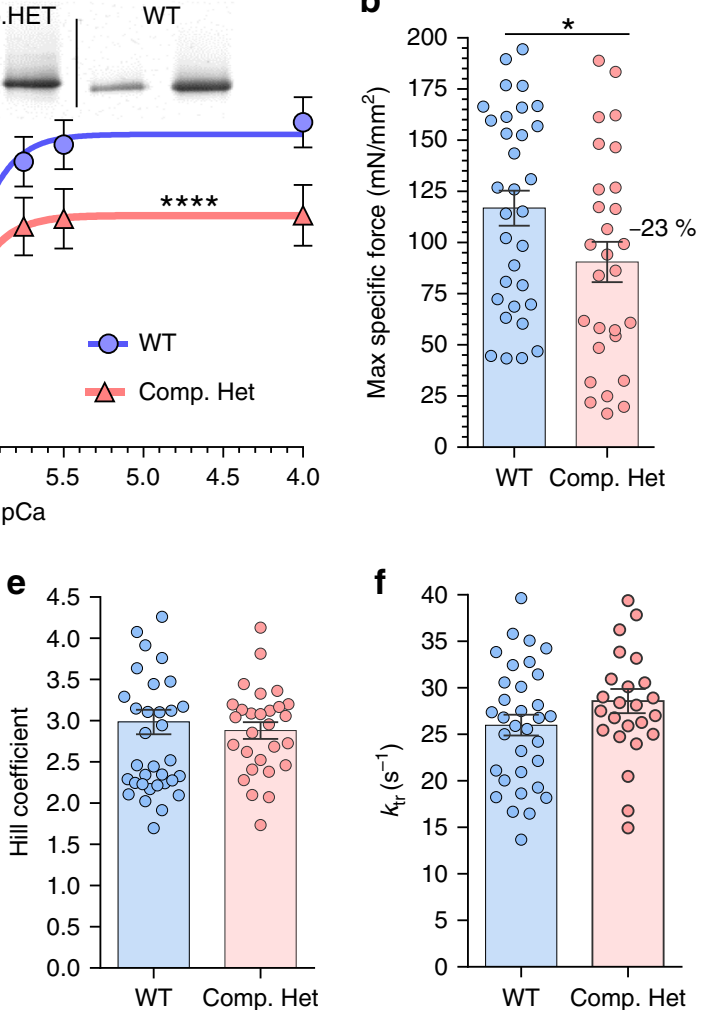

f

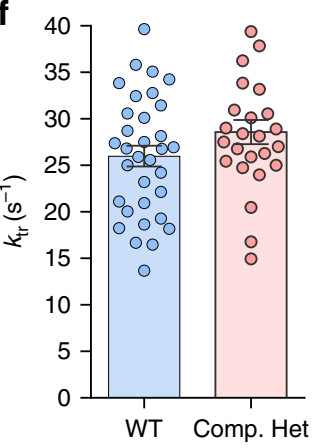

C

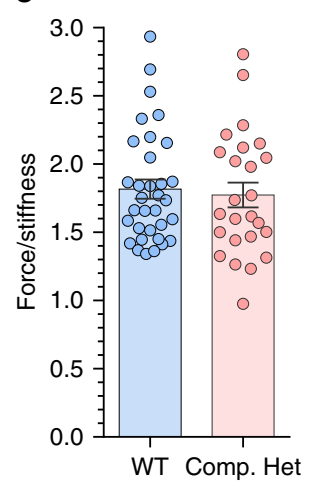

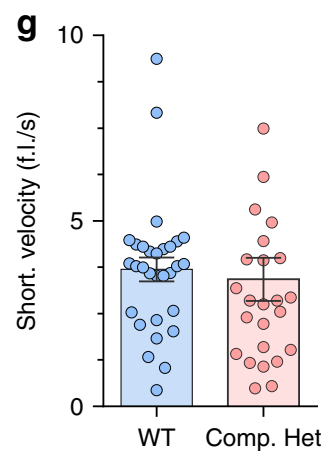

Fig. 7 Single fiber mechanics in type IIB fibers (EDL muscle) of Compound-Het mice. a Top: MHC-gel. Examples of IIB single fibers that were used in experiment. Two Compound-Het and two WT fibers are shown and a total of 41 WT and 40 Compound-Het fibers were studied; (WT: $34 / 41$ fibers were IIB; Compound-Het: 28/40 fibers were IIB). The left lane is a standard with all MHC isoforms. a Bottom: Specific force -pCa relations of Compound-Het and WT fibers. A Hill-type curve-fit analysis shows that the Compound-Het fibers are significantly different from WT fibers. $\mathbf{b}$ Maximal specific force is reduced in Compound-Het fibers $(p=0.046)$. c The force/stiffness relation is unaltered. Increase in calcium sensitivity $(p=0.028)(\mathbf{d})$ but unchanged cooperativity (e) in Compound-Het fibers. (Values based on force-pCa curves that were normalized to the maximal specific force.) Kinetics of tension redevelopment as reflected in $k_{\mathrm{tr}}(\mathbf{f})$ and maximal shortening velocity $(\mathbf{g})$ are unaltered in Compound-Het fibers. The data set comprises on average 7 type IIB fibers from each of 5 WT mice and 5 type IIB fibers from each of 6 Compound-HET mice. Values are mean \pm SEM. a: allosteric sigmoidal non-linear least squares fit with as null hypothesis that one curve fits all data sets. Test (Sum-of-squares F-test) reveals that the null hypothesis is rejected ( $p \leq 0.0001$, F (DFn, DFd) $8.146(3,360)$ ). b-g two-tailed unpaired t-test. ${ }^{*} p<0.05 ;{ }^{* \star \star \star} p<0.0001$. Source data are provided as a Source Data file.

active stiffness, rate constant of force redevelopment $\left(k_{\mathrm{tr}}\right)$ and maximum unloaded shortening velocity were measured at a sarcomere length of $2.5 \mu \mathrm{m}$. Afterward, the fiber was recovered, and its myosin isoform was determined. As fibers from WT and Compound-Het mice were mostly type IIB, analysis was restricted to this fiber type (Fig. 7a, top). We observed a $23 \%$ reduction in maximal specific-force generated by Compound-Het fibers (Fig. 7a, bottom and Fig. 7b). Active stiffness is a measurement of the number of force-generating cross-bridges and series compliance. No change in stiffness or the force/stiffness ratio was observed when comparing Compound-Het and control fibers (Fig. 7c). Interestingly, a small leftward shift in the force-pCa relationship was detected in Compound-Het fibers due to increased calcium sensitivity while cooperativity was unaffected (Fig. $7 \mathrm{~d}, \mathrm{e}$ ). The $k_{\mathrm{tr}}$ was determined by rapidly shortening and restretching of the fiber and fitting an exponential curve to the force redevelopment curve. $k_{\text {tr }}$ was not different between genotypes (Fig. 7f). Finally, the unloaded shortening velocity, measured by performing the slack test procedure ${ }^{50}$, was maintained in fibers from Compound-Het mice (Fig. $7 \mathrm{~g}$ ). Thus, Compound-Het fibers have a reduced maximal specific-force whereas calcium sensitivity is increased and the kinetic parameters are unaltered.

$\mathrm{X}$-ray diffraction reveals thin filament structural alterations. Small-angle X-ray diffraction is a powerful technique to study the structural alterations caused by mutations in myofilament proteins, under physiological conditions in intact muscle. The EDL muscle was studied in its passive state before activation, and during the plateau of a tetanic contraction in Compound-Het and WT mice. A schematic of the setup and typical X-ray diffraction images are in Fig. 8a, b. We focused on thin filament-based reflections and first determined the stiffness of the thin filaments, using the $27 \AA$ meridional reflection that arises from the actin subunit spacing. The spacing of the $27 \AA$ reflection during the tetanic force plateau was increased by $\sim 0.3 \%$ in both WT and Compound-Het muscles (Fig. 8c). The stiffness of thin filaments was determined from the $27 \AA$ spacing change and by converting the tetanic force of the whole muscle to force per thin filament ${ }^{30}$, as explained in Supplementary Fig. 6. Stiffness values so obtained were identical in WT and Compound-Het muscles, $33.4 \pm 3.5$ and $33.6 \pm 2.4 \mathrm{pN} / \mathrm{nm} / \mu \mathrm{m}$ (Fig. $8 \mathrm{~d}$ ). Unlike in nebulin deficient muscle where the thin filament stiffness is 3 -fold reduced $^{30}$, thin filament stiffness is unaltered in the Compound-Het model.

The thin filament helix was also studied, by analyzing the 6th and the 7th actin layer lines (ALL6 and ALL7), which occur at $\sim 59 \AA$ and $\sim 51 \AA$, respectively. The $51 \AA$ spacing was unchanged (Fig. 8e, left) but, interestingly, the $59 \AA$ spacing was significantly reduced (Fig. 8e, right). The ALL6 also had a reduced intensity (see Supplementary Fig. 6c). These findings suggest that the thin filament helix is twisted relative to WT in the Compound-Het. 
a

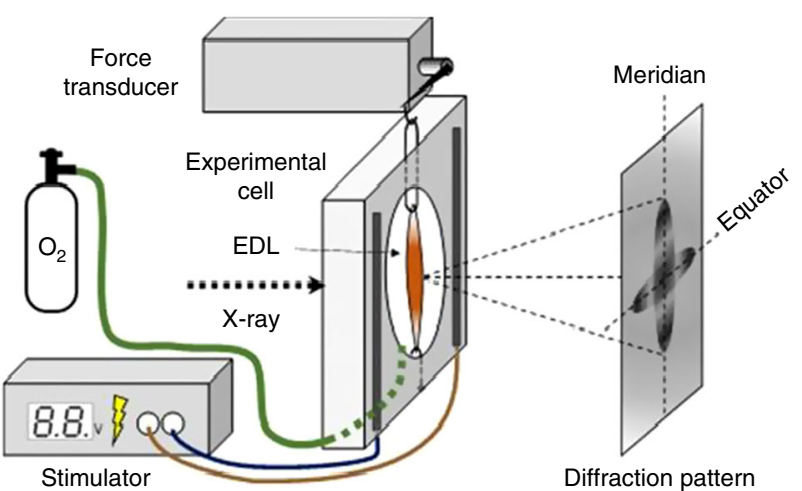

b

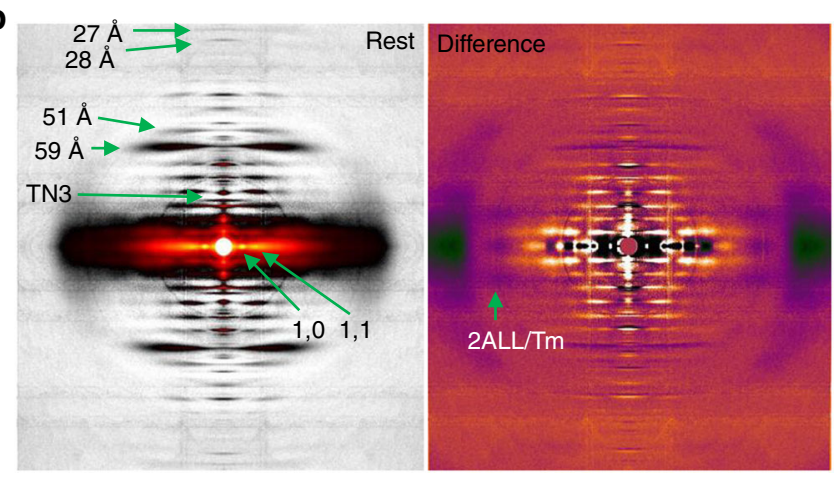

e

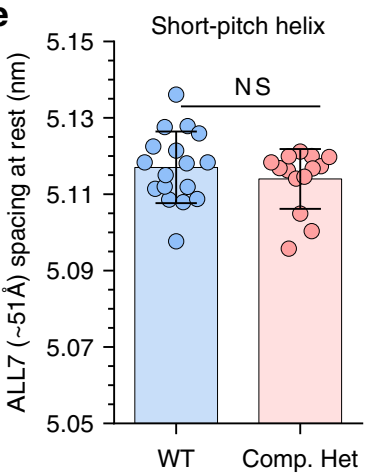

g

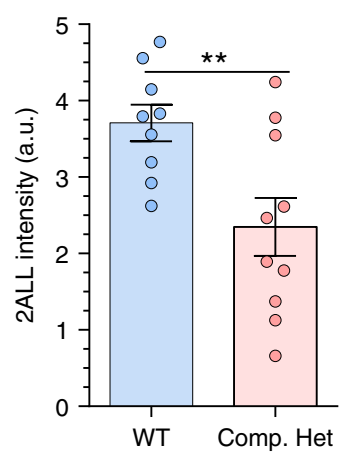

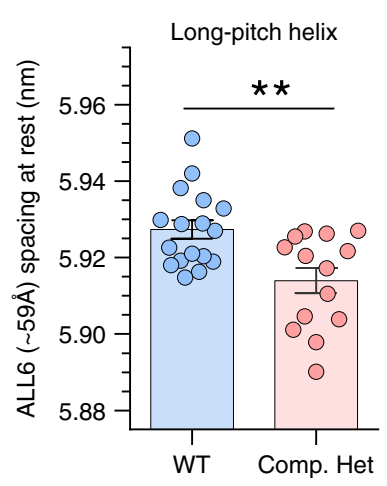

h

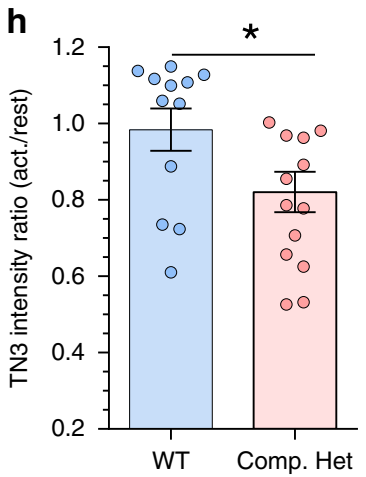

C

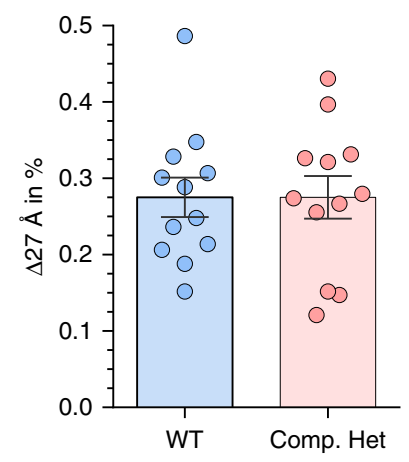

d

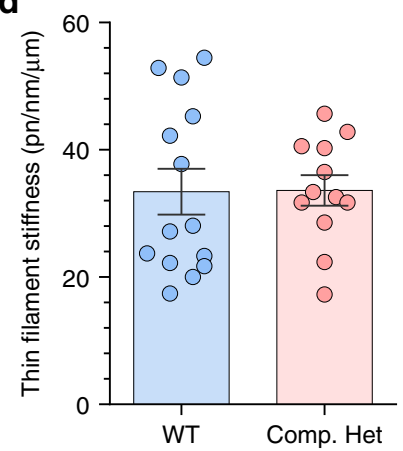

f

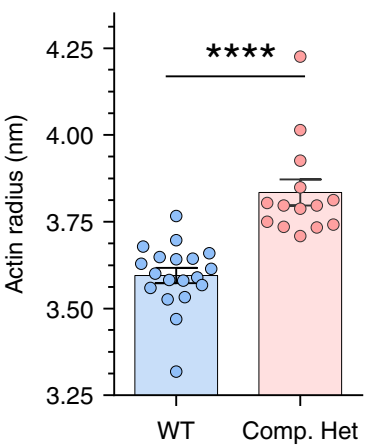

i

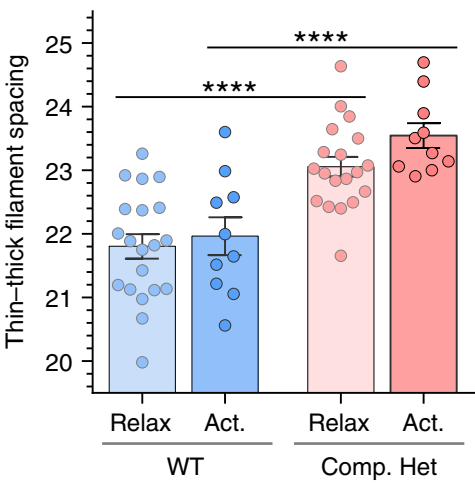

To determine if this twist has an effect on the actin radius, the radial separation of the intensity maxima of ALL6 was measured and observed to be significantly decreased in the Compound-Het, indicating an increased actin filament radius in the CompoundHet (Fig. 8f).

To determine whether the alteration in the long-pitch actin helix affects tropomyosin and troponin behavior, the off-meridional second actin layer line (2ALL (tropomyosin)) and the meridional third-order troponin (TN3) reflections were analyzed. Significantly less intense 2ALL and TN3 reflections during tetanic contraction were found in the Compound-Het EDL (Fig. 8g, h). Thus, it appears that structural changes in the actin helix caused by the nebulin mutation also alter the behavior of troponin and tropomyosin during contraction. 
Fig. 8 Thin filament structure assessed by X-ray diffraction on intact EDL muscle. a Experimental setup. $\mathbf{b}$ X-ray diffraction images with examined reflections indicated. Left image taken during rest and Right image is the difference between active and passive muscle. (See Methods for details.) $\mathbf{c}$ The spacing change of the 13th actin meridional reflection ( $27 \AA$ ), during tetanic force development, is identical in both genotypes. $\mathbf{d}$ Thin filament stiffness is identical in both genotypes (for calculations, see Supplementary Fig. 6b, c and Results). e Short(left) - and long(right)-pitch helical periodicity of the actin filament. The long-pitch helix (right) is significantly reduced $(p=0.0025)$ in the Compound-Het. $\mathbf{f}$ The actin radius, determined from the radial spacing of the ALL6, is increased in Compound-Het. $\mathbf{g}$ The intensity change of the second actin layer line (due to tropomyosin) and $\mathbf{h}$ the intensity of the third meridional troponin reflection upon activation, are both reduced in the Compound-Het EDL muscle $(P=0.0089$ and $p=0.044$., respectively). $\mathbf{i}$ Thin-thick filament spacing $(2 / 3 \times \mathrm{d} 1,0)$ is increased in compound Het, both in the relaxed and active states. (Based on d1,0 data in Supplementary Fig. $6 a)$ Number of mice: WT: 15, Compound-HET: 11. For some animals both EDL muscles were used. Number of muscles: WT: 18, Compound-HET: 14. Bars and lines are mean \pm SEM. c-h two-tailed unpaired t-test. ${ }^{\star} p<0.05 ;{ }^{* \star} p<0.01 ;{ }^{* \star \star \star} p<0.0001$. $\mathbf{h}$ Ordinary one-way ANOVA without matching or paring. A posthoc multiple testing comparison with multiple testing correction (Tukey) was performed. ${ }^{\star \star \star \star} p<0.0001$. Source data are provided as a Source Data file.

Finally, the increased actin radius in Compound-Het muscles might affect myofilament lattice spacing, therefore we determined the thin-thick filament distances. This revealed that the distance between thin and thick filaments were $\sim 1.4 \mathrm{~nm}$ larger in both passive and active Compound-Het muscles (Fig. 8i).

\section{Discussion}

Mutations in nebulin are the most common genetic cause of NM, and most often present as typical NM, i.e., congenital muscle weakness that is not or only slowly progressive with a relatively normal life $\operatorname{span}^{3}$. Most disease-causing NEB mutations are compound-heterozygous with a missense mutation on one NEBallele and a more disruptive mutation on the other ${ }^{3,4,9}$. Many of the existing nebulin-based mouse models do not mimic mutations in patients but instead were created to study the basic functions of nebulin $21,22,27,29,51$. An exception is the $\mathrm{Neb}^{\Delta \text { ex55 }}$ model $^{35}$ that mimics NM patients with an in-frame deletion of exon $55^{41}$. However, homozygous $\mathrm{Neb}^{\Delta \mathrm{ex} 55}$ mice have a more severe phenotype than $\mathrm{Neb}^{\Delta \mathrm{ex} 55}$ patients, as shown by their survival of less than a few days and near-complete disappearance of nebulin protein ${ }^{35}$, whereas homozygous $\mathrm{Neb}^{\Delta \mathrm{ex} 55}$ patients survive to adulthood and express $20-50 \%$ of control levels of nebulin protein ${ }^{9,42}$. Thus, existing mouse models do not represent nebulin-based typical NM. Here we successfully created a compound-heterozygous mouse that carries the Neb exon 55 deletion and the p.Ser6366Ile missense mutation, both identified as a myopathic founder mutation with world-wide occurrence ${ }^{36-38}$.

Existing nebulin models and patients with severe NM have reduced nebulin expression and shorter thin filaments ${ }^{21,22,26,27,42}$, a reduced $k_{\text {tr }}$ and lower calcium sensitivity $23,27,42,46$, and reduced force per crossbridge ${ }^{31}$. None of these changes are present in the Compound-Het where nebulin expression is unaltered (Fig. 3b), thin-filament length is slightly longer (Fig. 5d, Supplementary Fig. $4 \mathrm{~d}$ ), $k_{\mathrm{tr}}$ is normal and calcium sensitivity is increased (Fig. $7 \mathrm{~d}$, f). Despite the normal levels of nebulin, NM characteristics are clearly present (e.g., nemaline rod bodies, muscle weakness, muscle atrophy, etc.). Additionally, a fiber-type shift toward slower myosin isoforms occurs in Compound-Hets (Fig. 4), as seen in patients with $\mathrm{NM}$ and existing nebulin mouse models $8,27,52$. Hence, factors other than nebulin content per se can cause nebulinbased NM, and typical and severe NM have different disease mechanisms.

The nebulin protein expressed in the Compound-Het model could be transcribed from either Neb-allele. Crossing the Compound-Het or the $\mathrm{Neb}^{\mathrm{S} 6366 \mathrm{I}}$ to a model that expresses a smaller nebulin $\left(\mathrm{Neb}^{\Delta \mathrm{S} 9-11}\right)$ provides evidence that the Neb$\Delta$ Exon55-allele does not produce protein and that the $\mathrm{Neb}^{\text {S6366I }}$ allele does produce nebulin (Fig. 3a). It is therefore likely that the Compound-Het mice express mainly nebulin with the S63661I substitution. The Compound-Het mouse has a more severe phenotype than the $\mathrm{Neb}^{\mathrm{S} 6366 \mathrm{I}}$ Hom model (e.g., Figs. 1e, f, 2, 5, and 6) even though both models are likely to express exclusively nebulin with the S63661I substitution and, furthermore, both models express nebulin at normal WT levels (Fig. 3b, c). This indicates that the Compound-Het is somehow affected by the

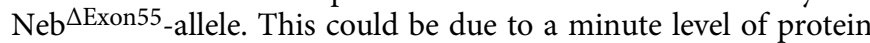

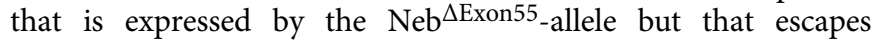
detection in the protein gel assays. For example, $\Delta$ Exon55nebulin might not be stable and degrade easily and, therefore, not show up as a full-length protein, with some of the degradation product functioning as a poison-peptide. In addition, the loss of $\mathrm{Neb}^{\Delta \text { Exon} 55}$ transcript, possibly due to mRNA-mediated decay ${ }^{35}$, could cause cellular stress that exacerbates the phenotype in Compound-Het mice. An alternative explanation for the more severe phenotype of the Compound-Het is that a single $\mathrm{Neb}^{\text {S6366I }}$-allele is unable to supply enough nebulin to accommodate the normal rate of protein turnover. Consequently, the S6366I protein might have a longer half-life in the CompoundHet than in the $\mathrm{Neb}^{\text {S6366I }} \mathrm{Hom}$, and perhaps accumulate additional post-translational modifications that are deleterious. Understanding how a single allele with a missense mutation produces a more severe myopathy compared to a homozygous state with the missense mutations warrants future studies. Considering that typical NM patients often are compound heterozygous with one nonsense and one missense $N E B$ mutation ${ }^{3,4,9}$, these future studies have high clinical relevance.

Nebulin comprises 25 super-repeats, each containing 7 SDxxYK actin-binding motifs ${ }^{18}$. The super-repeats at the ends of the molecule were recently shown to bind several-fold more strongly to actin in vitro than the central super-repeats ${ }^{53}$. The $\mathrm{p}$. Ser6366Ile mutation is found in super-repeat 18 (or 22 in the mouse) which supposedly binds weakly to actin. Interestingly, the Ser6366Ile mutation strengthens the actin-binding affinity of nebulin $^{39}$. The muscle weakness of both the Compound-Het and $\mathrm{Neb}^{\text {S6366I }}$ Hom models suggests that it is important to have weakbinding super-repeats in the central region of nebulin.

Initially we considered the possibility that the S6366I mutation alters thin filament stiffness and X-ray diffraction studies were conducted to measure the extension of the actin subunit spacing (27 $\AA$ reflection) during activation. By combining this measurement with the measured tetanic force, scaled down to force per thin filament, a thin filament stiffness value of $33.6 \mathrm{pN} / \mathrm{nm} / \mu \mathrm{m}$ in Compound-Het muscle is obtained which is indistinguishable from the $33.4 \mathrm{pN} / \mathrm{nm} / \mu \mathrm{m}$ in $\mathrm{WT}$ muscle. Thus, it is unlikely that altered thin filament stiffness plays a role in the phenotype of the Compound-Het mouse.

An earlier image reconstruction study reported evidence for multiple nebulin binding sites on the outer domains of each actin subunit $^{54}$. Furthermore, it has been shown that the presence of nebulin facilitates tropomyosin movement during tetanic contraction $^{30}$. These studies support the notion that during contraction, nebulin moves on the actin filament, in conjunction with tropomyosin. It is conceivable that such movement requires weak 
binding between nebulin and actin and, thus, an increase in actinbinding affinity (Ser6366Ile mutation) might be deleterious.

The X-ray diffraction study revealed changes in the F-actin structure in the Compound-Het as shown by the significantly reduced $\sim 59 \AA$ spacing and unaltered $\sim 51 \AA$ spacing (Fig. 8 e). The spacings of the $59 \AA$ and $51 \AA$ layer lines correspond to the pitches of the left-handed and right-handed one-start helices of the F-actin filament, respectively (e.g., see Fig. 2a of Volkmann et al. ${ }^{55}$ ). These $59 \AA$ and $51 \AA$ layer line spacings are known to slightly increase during isometric contraction and decrease during unloaded shortening, reflecting changes in thin filament strain ${ }^{56}$. However, they do not behave identically, during neither isometric contraction nor unloaded shortening, reflecting changes in thin filament twist ${ }^{56}$. Importantly, we previously found in the nebulin cKO mouse that the $59 \AA$ spacing is reduced without affecting the $51 \AA$ spacing nor the baseline actin monomer axial spacing (27 $\AA$ ), revealing that nebulin increases the thin filament helical twist $^{30}$. The findings of the present work in the Compound-Het model (unaltered $51 \AA$ spacing, reduced $59 \AA$ spacing, no change in actin monomer spacing) are consistent with this observation and support that nebulin plays a role in the twist of the F-actin filament. Our finding that the thin filament diameter is slightly but significantly increased (Fig. $8 \mathrm{f}$ ) likely reflects, at least in part, the increased helical twist. Bordas et al. ${ }^{56}$ developed an analytical expression for calculating the degree of twist and its direction using the differential spacing changes of the $51 \AA$ and $59 \AA$ reflections (for details, see ${ }^{56}$ ). This approach was used here (Fig. 8e) to calculate in Compound-Het muscle the degree of Factin helical twist, defined in terms of $\Delta n / n$ where $n$ is the number of subunits per turn of the actin double helix. A $0.9 \%$ increase in actin filament twist was found in the Compound-Het EDL muscles as compared to WT. This is accomplished with no significant change in the $27 \AA$ axial repeat of the subunits (Fig. 8c) consistent with a pure rotation of the subunits around the long axis.

Although the changes in the structure of F-actin in Compound-Het mice are small they nevertheless might have important consequences for thin filament activation and actomyosin interaction. Consistent with this possibility, we found altered tropomyosin and troponin behavior in the CompoundHet, as indicated by the reduced intensity of the 2ALL and the TN3 reflections during contraction (Fig. 8g, h). This might underlie the altered calcium sensitivity of skinned fibers (Fig. 7d) and contribute to the left-shift in the force-frequency relation of intact muscle (Figs. 1g, 6b, c). These changes, however, are unlikely to cause disease, as they will increase force at submaximal stimulation rates. It is worth pointing out, however, that increased calcium sensitivity is also expected to slow down the speed of relaxation, as was found in intact EDL and soleus muscle (Fig. 6f, Supplementary Fig. 5f), and that this might result in loss of functionality during rapid movements.

In addition, the X-ray diffraction studies revealed a $\sim 1.4 \mathrm{~nm}$ larger spacing between the thin and thick filaments (Fig. 8i) which is expected to decrease the number of myofilaments per unit cross-sectional area of myofibril by $12.9 \%$ (Supplementary Fig. $6 \mathrm{~b}$ ) and, hence, decrease specific force by $12.9 \%$. Considering the constant volume behavior of intact skeletal muscle ${ }^{57}$ the increased lattice spacing of the Compound-Het muscle could, in principle, be due to a $12.9 \%$ shorter sarcomere length. Sarcomere length was measured in passive muscle at $\mathrm{L}_{0}$ in a subset of the muscles used for X-ray diffraction (4 WT and 6 Compound-Het muscles) and the values obtained were indistinguishable $(2.76 \pm$ $0.08 \mu \mathrm{m}$ in WT and $2.69 \pm 0.04 \mu \mathrm{m}$ in Compound-Het; $p=0.41$ ). Furthermore, thin filament length was slightly increased in Compound-Het (Fig. 5d), indicating no underlying structural cause for decreased sarcomere length in Compound-Het muscles.
Could the expanded myofilament lattice be due to the structural effect of the Ser6366Ile mutation on the thin filament? The factors that determine lattice spacing are complex, and include thin and thick filament charges that cause electrostatic repulsive forces between filaments as well as lateral forces due to titin ${ }^{58}$, crossbridges and Z-disk and M-band structures ${ }^{57}$. We speculate that the twist and increased radius of the actin filament in the Ser6366Ile mouse result in altered charge distributions on the thin filament and that this contributes to the lattice expansion. Due to the complexity of the factors involved in setting the myofilament lattice spacing, future studies focused on the myofilament lattice are warranted.

A second factor that will affect the specific force of EDL muscle in the Compound-Het is the reduced fraction of the fiber crosssection that is taken up by myofibrils, 0.766 in Compound-Het vs 0.801 in WT $(p<0.01)$. This is expected to lower force by $\sim 3.5 \%$. This reduced fractional area is explained by the slightly higher fractional area of mitochondria in the Compound-Het muscles (Supplementary Fig. 6b). The reduced myofibrillar area and the above discussed myofilament lattice expansion add up to an expected specific-force deficit of $16.4 \%$, a considerable portion of the $23 \%$ force deficit in EDL fibers. In addition, wavy, misaligned and damaged Z-disks were found during ultrastructural examination of Compound-Hets (Fig. 5), which likely contributes to the muscle weakness. Finally, atrophy (13\% in EDL muscle) will reduce the total cross-sectional area of muscle which is expected to further lower the total force produced, in addition to the reduced specific force.

To summarize, we created and extensively characterized a typical NM mouse model with compound heterozygous mutations that mimic those found in NM patients. Compound-Het mice are growth-retarded, have atrophic muscles that contain nemaline rods, and have muscle weakness. Structural studies show that the thin filaments are slightly twisted and compared to wildtype thin filaments, tropomyosin and troponin behavior during activation is altered. Furthermore, the myofilament lattice spacing is increased and the fractional area of myofibrils is reduced. The Compound-Het myopathy takes place while nebulin expression is normal, thin filament lengths are slightly increased and crossbridge kinetics are unaltered. This contrasts with nebulin-based severe NM (e.g., $\mathrm{Neb}^{\Delta \mathrm{ex} 55}$ ) where nebulin expression is severely reduced, thin filaments are shorter, calcium sensitivity is reduced, and the force per cross-bridge and the fraction of force-generating cross-bridges are reduced, in mice $^{21,27,31}$ and patients ${ }^{42,46,47}$. Thus, the present study highlights the importance of understanding the different disease mechanisms that cause typical and severe NM, and that it is not possible to extrapolate from one to the other. Future studies are required to establish whether patho-mechanisms at play in patients with typical NM are similar to those in the CompoundHet mouse model. The $\mathrm{Neb}^{\mathrm{S} 6366 \mathrm{I}}$ Hom model has a phenotype that develops with age and appears to share similarities with Finnish distal myopathy patients ${ }^{36}$. The mechanistic correspondence between this model and patients also warrants further research. The models that were developed in this work will be useful for deciphering the pathophysiological mechanisms of typical NM and Finnish distal myopathy and for developing therapeutic approaches, to increase muscle strength, and bring much needed relief.

\section{Methods}

Generation of Compound-Het mouse model. The generation of $\mathrm{Neb}^{\Delta \mathrm{Exon} 55}$ has been described elsewhere ${ }^{35}$. To create the $\mathrm{Neb}^{\text {S6366I-mouse, a targeting }}$ vector to Knock-In the Ser -> Ile missense mutation was constructed with the Osdd vector which allows thymidine kinase as a negative selection cassette. (Note that this mutation was initially designated p.Ser4665Ile, but has been re-designated 
p.Ser6366Ile (www.lovd.nl/NEB.).) Overlapping primers introduced two nucleotide changes, one change created an MfeI restriction site (to facilitate distinguishing the targeted Knock-in and endogenous transcripts as well as allowing design of allelespecific PCR primers) and the other change resulted in the Ser $->$ Ile missense mutation corresponding to $\mathrm{c} .19097 \mathrm{G}>\mathrm{T}$ found in humans $\mathrm{s}^{9,36}$. Linearized targeting vector was electroporated into 129S6/SvEvTac cells; 6 G418-resistant ES cell clones were confirmed to have correctly integrated into the Neb gene. The clones were sequenced to verify the introduction of the mutation. The cells were injected into C57BL/6J blastocysts before transfer to recipient hosts. Chimeras were bred with C57BL/6J mice and neo-containing mice were identified via PCR. Heterozygous mice were then bred to a separate C57BL/6J mouse line containing a FLPase deleter to remove the neomycin cassette. Mice were backcrossed for ten generations before experiments were performed. The following primers were used for determining genotype after neo-removal and back crossing; exon55_WT_F2: 5'-GCATTCTT GCTCTTTCTTGTATGG-3', DeltaN_KO F: 5'-ACACCCAGGCAGAAGCTA GG-3', exon55 R: 5'-GAAAGGAACTCTGTCCTCTGG-3', NebEx106KI_F: 5'-AT CGTCATCTTGGCTTGGTT-3', NebEx106KI_R: 5'-TGTCTTTTTCCCTCCAA ACG-3'. Compound heterozygous NebS6366I/ $\Delta$ exon55 mice were produced by breeding $\mathrm{Neb}^{\mathrm{S6366I}}$ Het mice with $\mathrm{Neb}^{\Delta \operatorname{exon} 55}$ Het mice, which had also had the neo cassette removed and were backcrossed to C57BL/6J for 10 generations. Note that each of the mutations introduced in the $\mathrm{Neb}^{563661 / \Delta \operatorname{exon} 55}$ mouse has been found in patients, but that this combination has not been detected in patients as of yet. Mice are maintained within a pathogen-free barrier facility with $14 \mathrm{~h}: 10 \mathrm{~h}$ light: dark cycle in clear cages with access to water and food provided ad libitum. Most studies used 4 months old mice, except the studies in Fig. 1e, $\mathrm{f}$ (age on Figure), Fig. 8 ( 2 mo old), and S2 and 3 (as indicated on Figure). Male mice were used in all studies. Some studies used female mice as well but no gender differences were found in any comparisons. All animal experiments were approved by the University of Arizona and the Illinois Institute of Technology Institutional Animal Care and Use Committees and followed the NIH Guide for the Care and Use of Laboratory Animals.

Dissection. Mice were anesthetized with isoflurane before being euthanized by cervical dislocation. Body weights were measured and the heart, the diaphragm, and the skeletal muscles of the lower limbs were dissected and weighed; tibia length (TL) were also measured and used for normalizing muscle weights. The muscles were frozen in liquid N2.

Grip strength. Grip strength measurements were performed according to TREATNMD-protocol (www.treat-nmd.org). The mouse gripped a horizontal steel mesh with its front paws while being lifted by its tail. It was gently pulled away at a constant speed until its grip was broken. All four limbs were also tested by a similar approach; the mouse was placed on the steel mesh and gentle pulled by its tail until it released its grip. Peak tensions (grams of force) were recorded on a digital force gauge (Chatillon Force Measurement DFEII, Columbus Instruments) as the mouse released its grip.

In-vivo muscle analysis of the gastrocnemius muscle complex. In-vivo muscle analysis for the gastrocnemius complex was conducted using male $\mathrm{Neb}^{\mathrm{S} 6366 \mathrm{I}} \mathrm{Hom}$ and Compound-Het mice. Mice were anesthetized using isoflurane and placed on the heated platform $\left(39^{\circ} \mathrm{C}\right)$ of the Aurora Scientific Mouse Muscle Physiology System (model 809B; Aurora Scientific, Inc., Aurora, Ontario, Canada). Hair was removed from the right hind-leg and the knee immobilized using a non-invasive clamp. The foot was secured to the footplate on the force transducer (300C series with dual-mode lever systems, Aurora Scientific) with adhesive tape and set at a $90^{\circ}$ angle. Needle electrodes were placed distal to the knee, just under the skin in close proximity to the tibial and sural nerves. Optimal needle placement and pulse phase for plantar flexion was established using $10 \mathrm{~Hz}$ tetanus stimulations at $40 \mathrm{~mA}$. Forces were measured in $\mathrm{mN}$ using ASI 610A Dynamic Muscle Control v5.3 software. Optimal current was determined using twitch forces measured every $10 \mathrm{~s}$. The isometric force-frequency relationship was measured at $1,10,20,30,40$, $60,80,100,125,150$, and $200 \mathrm{~Hz}$ using the same stimulation parameters as described for $10 \mathrm{~Hz}$ stimulations (see force-frequency sequence below). Maximal tetanic force was typically achieved at $150 \mathrm{~Hz}$. Tissue weights for the gastrocnemius complex (gastrocnemius, plantaris, and soleus) were used for force normalization.

Intact muscle mechanics. The intact muscle mechanics have been described previously $29,59,60$. In short, EDL and soleus muscles were carefully, but quickly, excised and silk suture loops (USP 4-0) were tied to each tendon. The muscle was attached to a stationary hook and a servomotor-force transducer connected to an Aurora Scientific $1200 \mathrm{~A}$ isolated muscle system and muscles were submerged in an oxygenated Krebs-Ringer bicarbonate solution at $30^{\circ} \mathrm{C}$ (in mM: $137 \mathrm{NaCl}, 5 \mathrm{KCl}, 1$ $\mathrm{NaH} 2 \mathrm{PO} 4 \cdot \mathrm{H} 2 \mathrm{O}, 24 \mathrm{NaHCO} 3,2 \mathrm{CaCl} 2 \cdot 2 \mathrm{H} 2 \mathrm{O}, 1 \mathrm{MgSO} \cdot 7 \mathrm{H} 2 \mathrm{O}$ and 11 glucose; $\mathrm{pH7}$.4). Optimal length $\left(\mathrm{L}_{0}\right)$ was found by first performing a tetanus to remove any slack in the sutures, allowing the muscle to recover, and then increasing length until twitch forces plateaued. Force-frequency relationship was determined by subjecting muscles to increasing stimulation frequencies (in Hz: 1, 10, 20, 40, 60, 80, 100 and 150 for soleus, with an additional 200 for EDL). Muscles were allowed to recover for $30,30,60,90,120,120,120$ and 120 s between subsequent stimulations. Force obtained (converted to $\mathrm{mN}$ ) was normalized to the physiological CSA (PCSA) through the following equation: PCSA $=$ mass $(\mathrm{mg}) /$ [muscle $\operatorname{density}\left(\mathrm{mg} / \mathrm{mm}^{3}\right) *$ fiber length $\left.(\mathrm{mm})\right]$. The physiological density of muscle is $1.056 \mathrm{mg} / \mathrm{mm}^{3}$ and fiber length was found utilizing a fiber length to muscle length ratio, 0.72 for soleus and 0.51 for EDL ${ }^{61}$

Histology. Cross sections $(8 \mu \mathrm{m})$ of isopentane-frozen muscles were taken midbelly and stained with modified Gomori trichrome using standard histochemical techniques. For Gomori trichrome staining, sections were stained in Harris haematoxylin (Richard Allen Scientific) for 5 min, rinsed in tap water, and then stained in Gomori trichrome solution (pH 3.4) for $10 \mathrm{~min}$. Following an additional rinse and differentiation in $0.5 \%$ acetic acid for $10 \mathrm{~s}$, sections were dehydrated and coverslipped using standard techniques. Light microscopic images were captured using an AxioCam MRc (Carl Zeiss, Thornwood, NY, USA).

Transmission electron microscopy (TEM). For ultrastructural analysis, stretched EDL and soleus muscle were fixed in a mixture of 3.7\% paraformaldehyde, $3 \%$ glutaraldehyde and $0.2 \%$ tannic acid in $10 \mathrm{mM}$ PBS, $\mathrm{pH} 7.2$ at $4{ }^{\circ} \mathrm{C}$ for $1 \mathrm{~h}$. The muscles were then rinsed for $15 \mathrm{~min}$ in PBS and a postfixation was performed in $1 \% \mathrm{OsO} 4$ in the same buffer for $30 \mathrm{~min}$. Subsequently, samples were dehydrated in an ethanol graded series, infiltrated with propylene oxide and transferred to a mixture of 1:1 propylene oxide:Araldite 502/Embed 812 resin (Epon-812, EMS), then to a pure Araldite 502/Embed 812 resin, and finally polymerized for $48 \mathrm{~h}$ at $60^{\circ} \mathrm{C}$. Longitudinal ultrathin sections $(80 \mathrm{~nm})$ were obtained with a diamond knife (Diatome) in a Reichert-Jung ultramicrotome and contrasted with $1 \%$ potassium permanganate and lead citrate. Images $(1792 \times 1792$ pixel $)$ were acquired in a TECNAI Spirit G2 transmission electron microscope (FEI, Hillsboro, OR) with a side-mounted AMT Image Capture Engine V6.02 (4Mpix) digital camera, operated at $100 \mathrm{kV}$. ImageJ (v1.49, NIH, USA) was used for analysis of nemaline rod body size and thin filament lengths. TEM is known to cause a small amount of filament shrinkage during sample preparation (most likely during the dehydration and embedding steps) and therefore it is to be expected that the thick filaments will be slightly shorter than in vivo and to vary slightly in length from experiment to experiment. Filament shrinkage has been previously examined using the thick filament axial repeats measured on micrographs and comparing conventional transmission EM methods with quick freeze/freeze substitution methods ${ }^{15}$. Based on this earlier work and consistent with recent super-resolution optical microscopy ${ }^{62}$ thick filaments can be assumed to be $1.6 \mu \mathrm{m}$ in length. Measured thick and thin filament lengths obtained with TEM were adjusted for shrinkage accordingly.

Super-resolution Structured Illumination Microscopy (SR-SIM). For SR-SIM studies WT and Compound-Het 4 mo old mice were used. Skinned EDL fiber bundles were stretched from slack at different degrees ( 20-60\%), embedded in $\mathrm{O}$ C.T compound and immediately frozen in 2-methylbutane precooled in liquid nitrogen. $4 \mu \mathrm{m}$ thick cryosections were then cut and mounted onto microscope slides. Tissue sections were permeabilized in $0.2 \%$ Triton X-100/PBS for $20 \mathrm{~min}$ at room temperature, blocked with $2 \% \mathrm{BSA}$ and $1 \%$ normal donkey serum in PBS for $1 \mathrm{~h}$ at $4{ }^{\circ} \mathrm{C}$, and incubated overnight at $4{ }^{\circ} \mathrm{C}$ with primary antibodies diluted in blocking solution. The primary antibodies included: a rabbit polyclonal antiTmod1 $(3.33 \mu \mathrm{g} / \mathrm{mL})$, rabbit polyclonal anti-ETmod (Tmod1, $3.33 \mu \mathrm{g} / \mathrm{mL})$ and a mouse monoclonal anti-titin Ti102 $(2.5 \mu \mathrm{g} / \mathrm{mL})$. Sections were then washed with PBS for $2 \times 30 \mathrm{~min}$ and incubated with secondary antibodies diluted in PBS for $3 \mathrm{~h}$ at room temperature. The secondary antibodies included: AlexaFluor-647 conjugated donkey anti-rabbit IgG (1:200, Abcam) and AlexaFluor-405 conjugated donkey anti-mouse IgG (1:200, Invitrogen). The sections were then washed with PBS for $2 \times 15 \mathrm{~min}$ and covered with number $1.5 \mathrm{H}$ coverslips (Bioscience Tools, CSHP-No1.5-24 $\times 60$ ) using ProLong Diamond (Thermo Scientific, Inc.). A Zeiss ELYRA S1 SR-SIM microscope was used with UV light and solid-state laser $(405 / 488 / 561 / 642 \mathrm{~nm})$ illumination sources, a $100 \times$ oil immersion objective $(\mathrm{NA}=1.46)$, and a sCMOS camera. Typical imaging was performed on a $49.34 \times$ $49.34 \mu \mathrm{m}^{2}$ area with $1280 \times 1280$ pixel dimensions. Typical image stacks comprising of 40 slices were acquired with $0.084 \mu \mathrm{m}$ Z-steps, five angles and five phases/angle for each slice. Image reconstruction and fluorescence intensity plot profile generation were performed with ZEN 2 software (Zeiss). Plot profiles of the antibody-labeled images were fit with Gaussian curves to determine the epitope peak position using Fityk 1.3.0 software. Thin filament length was determined from the Tmodl epitope positions across the Z-disk. A-band width was determined from the Ti102 epitope positions across the A-band ${ }^{49}$

Sample preparation and gel electrophoresis. Muscle samples were prepared following a well-documented protocol ${ }^{63}$. Briefly, tissues were pulverized to powder via glass Dounce tissue Homogenizers pre-chilled in liquid nitrogen. Tissue powder was allowed to equilibrate in at $-20^{\circ} \mathrm{C}$ for $20 \mathrm{~min}$ before a $50 \%$ glycerol $/ \mathrm{H} 2 \mathrm{O}$ solution with protease inhibitors (in mM: $0.04 \mathrm{E}-64,0.16$ leupeptin and $0.5 \mathrm{PMSF}$ ) and a urea buffer (in M: 8 urea, 2 thiourea, 0.050 tris- $\mathrm{HCl}, 0.075$ dithiothreitol, $3 \%$ SDS w/v and $0.03 \%$ bromophenol blue, $\mathrm{pH}$ of 6.8 ) were added in a 1:40:40, sample $(\mathrm{mg})$ : glycerol $(\mu \mathrm{L})$ : urea $(\mu \mathrm{L})$, ratio. The solution was mixed and incubated at $60^{\circ} \mathrm{C}$ for $10 \mathrm{~min}$ before being aliquoted and flash frozen in liquid nitrogen. 
Nebulin was visualized on $1.0 \%$ agarose gels, run at $15 \mathrm{~mA} /$ gel for $2 \mathrm{~h}$ and $50 \mathrm{~min}$ and stained with Coomassie blue, as described previously ${ }^{63-65}$. Even though no molecular weight markers for identifying nebulin exist, based on the location of the larger titin (at top of gel) and the smaller myosin (most abundant protein), nebulin can be easily identified. Its location on agarose protein gels has also been confirmed in previous work with full nebulin KO models22. Myosin heavy chain isoform analysis was performed as previously described ${ }^{27}$. Briefly, myosin heavy chain isoforms were separated using $8 \%$ acrylamide gels, run for $24 \mathrm{~h}$ at $15{ }^{\circ} \mathrm{C}$ and a constant voltage of $275 \mathrm{~V}$ and stained with Coomassie blue. Gels were scanned and analyzed with Image (v1.49, NIH, USA). MHC type I and IIB are well separated on gels but the IIA overlaps with IIX due to insufficient separation. We refer to this band as IIA/X. The identity of the bands was established by running standard lysates consisting of a mixture of EDL and soleus muscles lysates, containing all isoforms in a well-established order (i.e., IIA/X at top, IIB in middle and I at bottom, e.g., see Fig. 4a, left).

Fiber cross-sectional area analysis. Muscle fiber cross-sectional area (CSA) was analyzed as described in $\mathrm{Li}$ et al. ${ }^{27}$. EDL and soleus muscles were pinned after stretching slightly beyond slack length on cork and covered with OCT (TissueTek). The samples were then frozen in liquid nitrogen-cooled isopentane. Muscles were cut mid-belly and frozen in OCT-blocks. Six $\mu$ m thick sections were collected on glass slides and stored at $-20^{\circ} \mathrm{C}$ for no longer than 2 days (Microm HM550, Thermo Fisher). Slides were equilibrated at room temperature for $10 \mathrm{~min}$ while individual sections were demarcated with a hydrophobic barrier (Vector Laboratories, Burlingame, CA, USA). Then, samples were treated with $0.2 \%$ triton X-100 in phosphate buffered saline (PBS) for $20 \mathrm{~min}$ followed by a $1 \mathrm{~h}$ incubation with a blocking solution ( $2 \% \mathrm{BSA}, 1 \%$ normal donkey serum) in PBS at $4{ }^{\circ} \mathrm{C}$. Primary antibodies were then applied to the sections for an overnight incubation at $4{ }^{\circ} \mathrm{C}$ : laminin (1:400 rabbit L9393, Sigma-Aldrich), MHCI (1:75 IgG2b BA-F8, DSHB), MHCIIA (1:500 IgG1 SC-71, DSHB), MHCIIX exclusion (1:100 IgG1 BF-35, DSHB) and MHCIIB (1:50 IgM BF-F3, DSHB). Following primary antibody incubation, sections were washed with PBS twice for $30 \mathrm{~min}$. The matching secondary antibodies were then applied for $3-4 \mathrm{~h}$ at room temperature: polyclonal Alexa Fluor 488-conjugated goat anti-rabbit [1:500 IgG (H + L) A11008, Thermo Fisher], polyclonal Alexa Fluor 350-conjugated goat anti-mouse [1:500 IgG2b A211440, Thermo Fisher], polyclonal Alexa Fluor 350-conjugated goat anti-mouse [1:500 IgG1 A21120, Thermo Fisher] and polyclonal Alexa Fluor 594-conjugated goat anti-mouse [1:500 IgM (Heavy Chain) A21044, Thermo Fisher]. Postsecondary washes included two 30 min washes with PBS followed by two quick rinses with water. Images were collected using an AxioCam MRc (Carl Zeiss, Thornwood, NY, USA) ${ }^{35}$. CSAs were analyzed using the semi-automatic muscle analysis using segmentation of histology (SMASH ver.5) MATLAB (R2015b) application ${ }^{66}$. The MinFeret distance was measured since it reflects the fiber size but is relatively insensitive to cutting angle during cryosectioning ${ }^{67}$.

Single fiber mechanics. EDL muscles were membrane-permeabilized (skinned) overnight at $\sim 4^{\circ} \mathrm{C}$ in relaxing solution (in mM: 40 BES, 10 EGTA, $6.56 \mathrm{MgCl} 2$, 5.88 NaATP, 1 DTT, 46.35 K-propionate, 15 creatine phosphate, Ionic strength $180 \mathrm{mM}, \mathrm{pH} 7.0$ at $20^{\circ} \mathrm{C}$ ) containing $1 \%$ Triton X-100 and protease inhibitors (in mM: 0.01 E64, 0.04 leupeptin and 0.5 PMSF). Muscles were then washed thoroughly with relaxing solution and stored in 50\% glycerol/relaxing solution at $-20^{\circ} \mathrm{C}$. Single fibers were dissected and mounted using aluminum T-clips between the length motor (ASI 322C, Aurora Scientific Inc.), and the force transducer element (ASI 403A, Aurora Scientific Inc.) in a skinned fiber apparatus (ASI 802D, Aurora Scientific Inc.). Sarcomere length was set in passive fibers to $2.5 \mu \mathrm{m}$ using a high-speed camera and ASI 900B software (Aurora Scientific Inc. v4.196). Muscle fibers were activated in $\mathrm{pCa}\left(\mathrm{pCa}=-\log \left(\left[\mathrm{Ca}^{2+}\right]\right) 4.0\right.$ activating solution (in $\mathrm{mM}$ : $40 \mathrm{BES}, 10 \mathrm{CaCO} 3 \mathrm{EGTA}, 6.29 \mathrm{MgCl} 2,6.12 \mathrm{Na}$-ATP, 1 DTT, 45.3 potassiumpropionate, 15 creatine phosphate, Ionic strength $180 \mathrm{mM}, \mathrm{pH} 7.0$ at $15^{\circ} \mathrm{C}$ ) and protease inhibitors. Fiber width and depth (built-in prisms allow for side view of fibers and measurement of depth) were measured at four points along the fiber, and the cross-sectional area (CSA) was calculated assuming an elliptical cross-section. Specific force was expressed as force per CSA $\left(\mathrm{mN} / \mathrm{mm}^{2}\right)$.

Force-pCa curves, $k_{\mathrm{tr}}$ and maximal shortening speed: Fibers were initially in relaxing solution and then moved into pre-activating solution (relaxing solution with a 10 -fold lower EGTA concentration), followed by activation at $15^{\circ} \mathrm{C}$ with incrementally increased $\mathrm{pCa}(\mathrm{pCa}=-\log ([\mathrm{Ca} 2+])$, ranging from 6.5 to 4.0 . The obtained force-pCa relation was fitted with a Hill equation, providing $\mathrm{pCa}_{50}(\mathrm{pCa}$ giving $50 \%$ of maximal active force) and the Hill coefficient, $\mathrm{nH}$, an index of myofilament cooperativity. Active stiffness: Active stiffness is an estimate of the number of force-generating cross-bridges during activation and was measured by lengthening and shortening the maximal activated fiber with $0.3,0.6,0.9 \%$ and $-0.3,-0.6,-0.9 \%$ of initial fiber length. The resulting change in tension was plotted against the length change (in \%) and the slope of the linear relationship is the active stiffness. $k_{\mathrm{tr}}$-measurements: The rate of force redevelopment $\left(k_{\mathrm{tr}}\right)$ was measured at steady-state force by rapidly shortening $(1 \mathrm{~ms})$ the fiber by $30 \%$ at one end of the fiber resulting in unloaded shortening of the fiber for $20 \mathrm{~ms}$. Remaining bound cross bridges were detached by rapidly restretching the fiber to initial length and the force redeveloped ${ }^{68}$. The rate constants of the double-exponential force redevelopment curve $\left(k_{\mathrm{tr}}\right)$ was determined by fitting the rise of force to the following equation (two-phase association curve): $\mathrm{F}=\mathrm{Fss} *\left(\left(1-\mathrm{e}^{\mathrm{ktr} * \mathrm{t}}\right)+(1-\right.$ $\left.\left.\mathrm{e}^{-\mathrm{k}^{*} \mathrm{t}}\right)\right)+\mathrm{c}$, where $\mathrm{F}$ is force at time $\mathrm{t}$, Fss is steady state force, $\mathrm{k}$ is the constant of the slow phase of force redevelopment; $k_{\mathrm{tr}}$ is the time constant of the fast phase of force redevelopment (the fast phase accounts for $>90 \%$ of the response and $k_{\mathrm{tr}}$ is plotted in Fig. 7). Maximal shortening velocity: The fiber was activated and allowed to reach steady state force and when rapidly shortened by $8,9,10,11,13$ and $15 \%$ of initial length resulting in a brief period of unloaded shortening. The time to start of the force recovery was measured, and this time was plotted against the length change. The slope is the unloaded shortening velocity ${ }^{50}$.

After mechanical experiments, the fiber was stored in SDS-sample buffer $(15 \%$ Glycerol, 2\% SDS, $62 \mathrm{mM}$ Tris-HCl pH6.8, $2.5 \mathrm{mM}$ Bromophenol Blue) for gel electrophoresis and myosin heavy chain isoform determination using silver-stained myosin gels (as mentioned above). Fibers containing more than 95\% type IIB myosin of total myosin isoforms were considered as type IIB fibers.

X-ray diffraction experiments. X-ray diffraction images were recorded on intact EDL muscles with a similar approach as in Kiss et al. ${ }^{30}$ and Ma et al. ${ }^{69}$. Mice were killed by cervical dislocation during carbon dioxide anesthesia and the EDL muscles were quickly excised. Silk-sutures (USP 4-0) were tied to each tendon and the muscle was mounted in a custom-built chamber filled with oxygenated solution (in mM: $145 \mathrm{NaCl}, 2.5 \mathrm{KCl}, 1.0 \mathrm{MgSO} 4,1.0 \mathrm{CaCl} 2,10.0$ HEPES, 11 glucose; $\mathrm{pH} 7.4$ ) and kept at room temperature. The optimal length (L0) was determined by adjusting muscle length until maximal twitch force was produced. Force was measured with a combined motor/force transducer (Model 6350; Cambridge Technology, Bedford, MA) connected to a dual-mode controller (300C; Aurora Scientific, Aurora, Canada). Intact muscles were activated with a high-power biphasic current stimulator (model $701 \mathrm{~A}$; Aurora Scientific). The whole system was controlled by an ASI $610 \mathrm{~A}$ data acquisition and control system (Aurora Scientific). X-ray diffraction patterns were recorded using the high-flux $12 \mathrm{keV}$ $\mathrm{X}$-ray beam provided by Beamline 18 at the Advanced Photon Source (Argonne National Laboratory. The BioCAT undulator beamline 18ID is a facility for biological non-crystalline diffraction and X-ray absorption spectroscopy at the Advanced Photon Source ${ }^{70}$.). Raw image frames were collected using a Dectris Pilatus $31 \mathrm{M}$ (Dectris AG, Baden-Dättwil, Switzerland) photon counting detector with $981 \times 1043,172 \times 172 \mu \mathrm{m}$, pixels with a 20-bit dynamic range. Background images were recorded by passing the X-ray beam through the experimental cell just next to the muscle. For the mechanical and X-ray experiments, the muscles were activated by an $80 \mathrm{~Hz}$ pulse frequency, $2 \mathrm{~ms}$ pulse width stimulation for a $4.5 \mathrm{~s}$ isometric tetanic contraction. A $3 \mathrm{~s}$ exposure of inactive muscle was collected before activation and a $4.5 \mathrm{~s}$ exposure during the plateau of the tetanus with the detector continuously collecting data frames with a $10 \mathrm{~ms}$ exposure time and $10 \mathrm{~ms}$ readout time. The muscle was oscillated in the beam during the exposure to sample a large volume of the muscle and spread the X-ray dose and prevent radiation damage. After the experiment, a subset of EDL muscles was chemically fixed in $10 \%$ formalin at optimal length. Fiber bundles were dissected and their sarcomere length measured, using fiber bundles throughout the entire muscle volume.

The X-ray detector data were saved as 32-bit TIFF-images. The individual images from a muscle at rest were averaged together into a single image and the individual images taken during the tetanic force plateau were also averaged into a single image. The averaged images were analyzed using the MuscleX software (ver. 1.13.1) developed at BioCAT (https://musclex.readthedocs.io/en/latest/. J. Jiratrakanvong, J. Shao, M. Menendez, X. Li, J. Li, Weikang Ma. G. Agam, T. Irving MuscleX: software suite for diffraction X-ray imaging V1.13.1, https://doi.org/ 10.5281/zenodo.1195050, March 2018.) Briefly, the lattice spacing and equatorial intensities were analyzed by the "Equator" routine in MuscleX. The images were quadrant folded and background subtracted using the "Quadrant fold" routine in MuscleX and the diffuse background intensities were summed to be used as normalization standard (details in ${ }^{71}$ ). The spacings and intensities of meridional reflections and layer lines were measured using the "Projection Trace" routine in MuscleX. The troponin and tropomyosin reflections were analyzed as described previously ${ }^{30}$. The thin filament helical pitches were measured by the axial spacings of the $59 \AA$ and $51 \AA$ actin layer lines. The $59 \AA$ layer line (ALL6) is derived from the left-handed helix joining the G- actin subunits and the $51 \AA$ actin layer line (ALL7) from the right-handed helix. The intensity in the layer lines were projected onto a line parallel to the meridional axis in the reciprocal radial range of $0 \leq \mathrm{R} \leq$ $0.053 \mathrm{~nm}^{-1,72}$. The axial spacings of the layer lines were estimated from the centroid of the peaks. The intensity of the $59 \AA$ reflection was estimated by fitting the radial intensity projection of $59 \AA$ 年flection with a Gaussian function. The radial spacing of the first maxima on the $59 \AA$ reflection in reciprocal space was estimated by calculating the distance between the centroids of the first maxima of the radially projected intensity distributions of the $59 \AA$ layer line to the meridian. From the radial spacing $(r)$ one can derive the distance from the center of the thin filament to the center of mass of the actin subunit $(R)$ by assuming that the ALL6 can be modeled by a J1 Bessel function where the first maximum occurs when the Bessel function argument $2 \pi R r$ is equal to 1.85 . We define the thin filament radius to be the value of $\mathrm{R}$ derived from this relation.

\section{Statistical analysis}

General. All measurements were obtained from distinct samples. The shown bar graphs are mean \pm SEM. Statistical software used: Graphpad Prism 7. For 
significant differences, $\mathrm{P}$-values, $\mathrm{T}$-values or $\mathrm{F}$-values and Degrees of Freedom (DoF) are indicated. Symbols ${ }^{*} p<0.05 ;{ }^{*} p<0.01 ; * * * p<0.001 ; * * * * p<0.0001$

Statistical details. Figure 1c) Gompertz growth curves; goodness of fit: $\mathrm{R}^{2}$ in WT 0.94 in Compound-Het 0.95; Test: does one curve adequately fit the data? No, $P<$ 0.0001 , F-value: 63.24, DoF: 344 Number of mice: WT: 21 , Compound-Het: 18 , and S6366I Hom: 15. d) One-way ANOVA (no matching or pairing), corrected for multiple comparisons (Tukey). Number of mice: WT: 21, Compound-Het: 18, and S6366I Hom: 15. $P=0.004$, F-value: 6.184, DoF: 52. e) Two-way ANOVA reveals a significant effect of age $(p<0.0001 ; F=300, \mathrm{DoF}=119)$ and genotype $(p<0.0001$; $\mathrm{F}=19, \mathrm{DoF}=119)$ on grip strength but without interaction. A posthoc multiple testing comparison with multiple testing correction (Dunnett) reveals a significant grip strength reduction at all 3 ages in Compound-Het mice and a significant reduction in 10 mo old NebS6366I mice. WT 18,18, 12; Compound-Het 19, 23, 14; S6366I HOM 14, 18, 6 for $1,4,10$ mo old mice, respectively. ${ }^{*} p<0.05$; ${ }^{*} p<0.05$; $* * * p<0.001$. f) Left: Force-frequency fit: ( $\mathrm{Y}=$ MinForce + (MaxForce - MinForce $) /\left(1+(\text { HalfFreq } / \mathrm{X})^{\wedge}\right.$ Hill cooef.) $)$; goodness of fit: $\mathrm{R}^{2}$ in WT: 0.98 , in Compound-Het: 0.97, and in S6366I Hom: 0.97; Test: does one curve adequately fit the data? No, $P<0.0001$, F-value: 104, DoF: 216. Number of mice: WT: 9, CompoundHet: 7, and S6366I Hom: 6 mice. Middle: Force-frequency fit: $(\mathrm{Y}=$ MinForce + (MaxForce - MinForce) $/(1+$ (HalfFreq/X)^Hill coeff. $)$ ); goodness of fit: $\mathrm{R}^{2}$ in WT: 0.98, in Compound-Het: 0.99, and in S6366I Hom: 0.92; Test: does one curve adequately fit the data? No, $P<0.0001$, F-value: 18 , DoF: 68 . Number of mice: WT: 6, Compound-Het: 2, and S6366I Hom: 6 mice. Right: Force-frequency fit: (Y= MinForce $+($ MaxForce - MinForce $) /\left(1+(\text { HalfFreq } / \mathrm{X})^{\wedge}\right.$ Hill coeff. $\left.)\right)$; goodness of fit: $\mathrm{R}^{2}$ in WT: 0.96, in Compound-Het: 0.98, and in S6366I Hom: 0.96; Test: does one curve adequately fit the data? No, $P<0.0001$, F-value: 27 , DoF: 113 . Number of mice: WT: 6, Compound-Het: 4, and S6366I Hom: 3 mice. P-values at maximal force $(150 \mathrm{~Hz})$ for the 3 -genotypes calculated with one-way ANOVA and Dunnett's multiple comparisons test $\left(* p<0.05,{ }^{*} p<0.05,{ }^{*} * *<<0.001\right)$. The force deficit comparison in S6366I HOM mice of 3 and 10 mo was done with an unpaired twotailed T-tests $(p=0.04)$. G) Main panel and inset: One-way ANOVA (no matching or pairing); corrected for multiple comparisons (Tukey). $P=0.0239$, F-value: 12 , DoF: 21. Number of mice: WT: 9, Compound-Het: 7, and S6366I Hom: 6 mice.

Figure 2) 2Way ANOVA with multiple comparison (multiple comparison correction (Sidak)). a) EDL; $P=0.0003$, t-value: 4.09 . DoF: 27 , Plant; $P=0.0003$, t-value: 3.22, DoF; 27, Tib Cran: $P<0.0001$, t-value: 6.65, DoF; 27, Quad; $P<$ 0.0001 , t-value: 5.14, DoF; 27, Gast; $P<0.0001$, t-value: 10.22, DoF; 27 and Diaph; $P=0.01$, t-value: 2.74 , DoF; 26. N; WT: 13 ; Compound-Het 16. b) Plant; $P=$ 0.0002, t-value: 3.44, DoF; 21 and Gast; $P=0.005$, t-value: 3.11, DoF; 21 . N; WT: 7, S6366I Hom: 16 mice.

Figure 3) One-way ANOVA with multiple testing correction (no matching or pairing) and individual t-tests show no significant difference for any of the muscle types. Number of mice. B: WT: 9, Compound-Het: 8. Number of mice in C: WT: 7, and S6366I Hom: 7

Figure 4a) For each muscle type: Two-way ANOVA (factors: genotype and MHC isoforms) with multiple testing correction (Sidak). F-values for interaction; EDL: $P<0.0001$, F-value: 15.85, DoF: 39; Plant: $P<0.0001$, F-value: 52.58 , DoF; 42; Tib Cran: $P=0.0462$, F-value: 3.31, DoF; 42; Gast: $P=0.0007$, F-value: 8.68, DoF; 42 and Diaph: $P=0.0009$, F-value: 8.45 , DoF; 39 . Number of mice. B: WT: 9 , Compound-Het: 7.

Figure 4b) Two-way ANOVA (factors: genotype and fiber type) with multiple testing correction (Sidak). MinFerets for interaction effect; $P=0.0005$, F-value: 7.49 and DoF: 37. Fiber count for interaction effect; $P=0.0005$, F-value: 7.23 and DoF: 41. Number of mice. B: WT: 5, Compound-Het: 7 Fig. 4c) Two-way ANOVA (factors: genotype and fiber type) with multiple testing correction (Sidak).

MinFerets for interaction effect; $P<0.0001$, F-value: 22.05 and DoF: 33 . Fiber count for interaction effect; $P<0.0001$, F-value: 27.37 and DoF: 43 . Number of mice. B: WT: 6, Compound-Het: 7

Figure 5b) Unpaired two-tailed T-tests. Four mice per genotype used with 5 fibers per muscle examined. Figure 5c) Unpaired two-tailed T-tests. Four mice per genotype used with 5 fibers per muscle examined. Figure 5d) One-way ANOVA (no matching or pairing); corrected for multiple comparisons (Tukey). $P<0.0001$, F-value: 27.57, DoF: 141. Six mice per genotype used with 5 fibers per muscle examined.

Figure 6a) Force-frequency fit: $(\mathrm{Y}=$ MinForce $+($ MaxForce - MinForce $) /(1+$ (HalfFreq/X)^Hill cooef.)); goodness of fit: $\mathrm{R}^{2}$ in WT: 0.86 , in Compound-Het: 0.90, and in S6366I Hom: 0.92. Test: does one curve adequately fit the data? No, $P<0.0001$, F-value: 10, DoF: 400. b) Relative force-frequency fit: $(\mathrm{Y}=$ MinForce + (MaxForce - MinForce) $/\left(1+(\text { HalfFreq/X })^{\wedge}\right.$ Hill cooef. $\left.)\right)$; goodness of fit: $\mathrm{R}^{2}$ in WT: 0.98, in Compound-Het: 0.98, and in S6366I Hom: 0.99; Test: does one curve adequately fit the data? No, $P<0.0001$, F-value: 21 , DoF: 400 ;

Figure $6 \mathrm{a}$ inset and $6 \mathrm{c}-\mathrm{f}$ ). One-way ANOVA (no matching or pairing); corrected for multiple comparisons (Sidak). a) $P=0.001$, F-value:8.32 and DoF:41, c) $P=0.0017$, F-value:7.69 and DoF:38, d) $P<0.0001$, F-value:36.3 and DoF:39, E) $\mathrm{P}=0.0002$, F-value: 11.15 and DoF: 40 and f) $P=0.0001$, F-value:32.86 and DoF: 40 . Number of mice: WT: 20, Compound-Het: 11, and S6366I Hom: 11.

Figure 7a) Allosteric sigmoidal curve with as test: does one curve adequately fit the data? No, $P<0.0001$. F-value: 8.146 , DoF: 363 . Goodness of fit: $\mathrm{R}^{2}$ in WT: 0.60 , in Compound-Het: 0.44. b-g) Unpaired two-tailed T-tests. b) $P=0.0464$, T-value: 2.035 and DoF: 58, d) $P=0.0282$, T-value: 2.246 and DoF: 58 , On average, 7 type
IIB fibers from each of 5 WT mice and 5 type IIB fibers from each of 6 CompoundHET mice.

Figure 8c-h) Unpaired two-tailed T-tests. e) right panel $P=0.0025$, T-value:3.3, DoF:29; f) $P<0.0001$, F-value:5.9 DoF:31, g) $P=0.0089$, F-value:2.954, DoF:17 and h) $P=0.0439$, F-value:2.127, DoF:24. I) Two-way ANOVA (factors: genotype and activation) with multiple testing correction (Sidak). For genotype effect: $P<$ 0.0001, F-value:48.32, DoF:65. Number of mice: WT: 15, Compound-HET: 11. For some animals both EDL muscles were used. Number of muscles: WT: 18, Compound-HET: 14

Supplementary Fig. 2a-c) For each muscle type: Two-way ANOVA (factors: genotype and MHC isoforms) with multiple testing correction (Sidak). Number of mice. a-c) WT: 6, and S6366I Hom: 8.

Supplementary Fig. 4b) WT: $\mathrm{Y}=0.01220 * \mathrm{X}+1.016$ (slope not significant from zero); S6366I Hom: $\mathrm{Y}=0.008872 * \mathrm{X}+1.043$ (slope not significant from zero); Compound-Het: $\mathrm{Y}=0.0268^{*} \mathrm{X}+1.01$ (slope significant from zero: $P=0.005, \mathrm{~F}$ value:8.012, DoF:119). The slopes are not different $(p=0.39)$ but the elevation is ( $p<0.001 ; \mathrm{F}: 9.6 ;$ DoF:334). 4c) One-way ANOVA (no matching or pairing); corrected for multiple comparisons (Tukey). $P<0.0001$, F-value: 27.57, DoF: 141 Six mice per genotype used with 5 fibers per muscle examined. d left) WT: Y = $0.124^{*} \mathrm{X}+0.7332$ (slope $p<0.001$ ); Compound-Het: $\mathrm{Y}=0.1671^{*} \mathrm{X}+0.657$ (slope $p<0.001)$. The slopes are not different (p:0.2674) but the elevation of the lines is different ( $p<0.001, \mathrm{~F} 29.6, \mathrm{DoF}: 291)$. d right) Unpaired two-tailed t-test, $p=0.01$, F:2.11, DoF 90. e left) WT: $\mathrm{Y}=0.07^{*} \mathrm{X}+1.44$ (slope $p<0.001$ ); Compound-Het: $\mathrm{Y}=0.1168 * \mathrm{X}+1.302$ (slope $p<0.001)$. The slopes are different (p:0.02). e right) Unpaired two-tailed t-test, $p=0.98$, F:1.1, DoF: 59 . Number of mice: WT: 3 and Compound-Het: 3 .

Supplementary Fig. 5a) Force-frequency fit: $(\mathrm{Y}=$ MinForce + (MaxForce MinForce $) /\left(1+(\text { HalfFreq/X })^{\wedge}\right.$ Hill cooef. $\left.)\right)$; goodness of fit: $\mathrm{R}^{2}$ in WT 0.95 , in Compound-Het 0.92, and in S6366I Hom 0.83. b) Relative force-frequency fit: $(\mathrm{Y}=$ MinForce $+($ MaxForce - MinForce $) /(1+($ HalfFreq $/ \mathrm{X}) \wedge$ Hill cooef. $)) ;$ goodness of fit: $\mathrm{R}^{2}$ in WT 0.98, in Compound-Het 0.99, and in S6366I Hom 0.99; A inset and c-f). One-way ANOVA (no matching or pairing); corrected for multiple comparisons (Sidak). c) $P=0.0094$, F-value:5.664, DoF:27; d) $P=0.0423$, Fvalue:3.047, DoF:32 and f) $P<0.0001$, F-value:18.0, DoF:27. Number of mice: WT: 13, Compound-Het: 7, S6366I Hom: 8.

Supplementary Fig. 6a, c) Two-way ANOVA (factors: genotype and activation) with multiple testing correction (Sidak) a) For genotype effect: $P<0.0001, \mathrm{~F}$ value:43.32, DoF:55 and c) for genotype: $P<0.0001$, F-value:16.22, DoF:41. Number of mice: WT: 7, Compound-HET: 7. Number of muscles: WT: 10 Compound-HET: 10. b) Two-way ANOVA for 'Void', mitochondria and myofibrillar fractions analyzed on 9900x magnification images: For interaction effect: $P<0.0001$, F-value: 13.6, DoF: 129 . Number of mice: 5 WT and 5 compound Het mice were analyzed with 4 cross-sections each.

Reporting summary. Further information on research design is available in the Nature Research Reporting Summary linked to this article.

\section{Data availability}

All data generated or analyzed during this study are included in this published article (and its supplementary information files). The source data underlying Figs. 1c-g, 2a, b, $3 \mathrm{~b}, \mathrm{c}, 4 \mathrm{a}-\mathrm{c}, 5 \mathrm{~b}-\mathrm{d}, 6 \mathrm{a}-\mathrm{f}, 7 \mathrm{a}-\mathrm{g}, 8 \mathrm{c}-\mathrm{i}$, and Supplementary Figs. 2, 4-7 are provided as a Source Data file.

Received: 9 September 2019; Accepted: 6 May 2020; Published online: 01 June 2020

\section{References}

1. Shy, G. M., Engel, W. K., Somers, J. E., Wanko, T. \& Nemaline Myopathy., A. New congenital myopathy. Brain 86, 793-810 (1963).

2. Romero, N. B. \& Clarke, N. F. Congenital myopathies. Handb. Clin. Neurol. 113, 1321-1336 (2013).

3. Sewry, C. A., Laitila, J. M. \& Wallgren-Pettersson, C. Nemaline myopathies: a current view. J. Muscle Res Cell Motil. https://doi.org/10.1007/s10974-01909519-9 (2019).

4. Gonorazky, H. D., Bonnemann, C. G. \& Dowling, J. J. The genetics of congenital myopathies. Handb. Clin. Neurol. 148, 549-564 (2018).

5. Wallgren-Pettersson, C., Beggs, A. H. \& Laing, N. G. 51st ENMC international workshop: nemaline myopathy. 13-15 June 1997, Naarden, The Netherlands. Neuromuscul. Disord. 8, 53-56 (1998).

6. Ryan, M. M. et al. Nemaline myopathy: a clinical study of 143 cases. Ann Neurol. 50, 312-320 (2001).

7. Wallgren-Pettersson, C. Genetics of the nemaline myopathies and the myotubular myopathies. Neuromuscul. Disord. 8, 401-404 (1998).

8. North, K. N., Laing, N. G. \& Wallgren-Pettersson, C. Nemaline myopathy: current concepts. The ENMC International Consortium and Nemaline Myopathy. J. Med Genet. 34, 705-713 (1997). 
9. Lehtokari, V. L. et al. Mutation update: the spectra of nebulin variants and associated myopathies. Hum. Mutat. 35, 1418-1426 (2014).

10. Pelin, K. et al. Mutations in the nebulin gene associated with autosomal recessive nemaline myopathy. Proc. Natl Acad. Sci. USA 96, 2305-2310 (1999).

11. Donner, K., Sandbacka, M., Lehtokari, V. L., Wallgren-Pettersson, C. \& Pelin, K. Complete genomic structure of the human nebulin gene and identification of alternatively spliced transcripts. Eur. J. Hum. Genet 12, 744-751 (2004).

12. Pfuhl, M., Winder, S. J. \& Pastore, A. Nebulin, a helical actin binding protein. ЕМВО J. 13, 1782-1789 (1994).

13. Labeit, S. \& Kolmerer, B. The complete primary structure of human nebulin and its correlation to muscle structure. J. Mol. Biol. 248, 308-315 (1995).

14. Kazmierski, S. T. et al. The complete mouse nebulin gene sequence and the identification of cardiac nebulin. J. Mol. Biol. 328, 835-846 (2003).

15. Sosa, H., Popp, D., Ouyang, G. \& Huxley, H. E. Ultrastructure of skeletal muscle fibers studied by a plunge quick freezing method: myofilament lengths. Biophys. J. 67, 283-292 (1994).

16. Kruger, M., Wright, J. \& Wang, K. Nebulin as a length regulator of thin filaments of vertebrate skeletal muscles: correlation of thin filament length, nebulin size, and epitope profile. J. Cell Biol. 115, 97-107 (1991).

17. Wang, K. \& Wright, J. Architecture of the sarcomere matrix of skeletal muscle: immunoelectron microscopic evidence that suggests a set of parallel inextensible nebulin filaments anchored at the Z line. J. Cell Biol. 107, 2199-2212 (1988).

18. Labeit, S. et al. Evidence that nebulin is a protein-ruler in muscle thin filaments. FEBS Lett. 282, 313-316 (1991).

19. Jin, J. P. \& Wang, K. Nebulin as a giant actin-binding template protein in skeletal muscle sarcomere. Interaction of actin and cloned human nebulin fragments. FEBS Lett. 281, 93-96 (1991).

20. Ogut, O., Hossain, M. M. \& Jin, J. P. Interactions between nebulin-like motifs and thin filament regulatory proteins. J. Biol. Chem. 278, 3089-3097 (2003).

21. Bang, M. L. et al. Nebulin-deficient mice exhibit shorter thin filament lengths and reduced contractile function in skeletal muscle. J. Cell Biol. 173, 905-916 (2006).

22. Witt, C. C. et al. Nebulin regulates thin filament length, contractility, and Zdisk structure in vivo. EMBO J. 25, 3843-3855 (2006).

23. Chandra, M. et al. Nebulin alters cross-bridge cycling kinetics and increases thin filament activation: a novel mechanism for increasing tension and reducing tension cost. J. Biol. Chem. 284, 30889-30896 (2009).

24. Ottenheijm, C. A. et al. Sarcoplasmic reticulum calcium uptake and speed of relaxation are depressed in nebulin-free skeletal muscle. FASEB J. 22, 2912-2919 (2008).

25. Ottenheijm, C. A. \& Granzier, H. New insights into the structural roles of nebulin in skeletal muscle. J. Biomed. Biotechnol. 2010, 968139 (2010).

26. Ottenheijm, C. A. et al. Altered myofilament function depresses force generation in patients with nebulin-based nemaline myopathy (NEM2). J. Struct. Biol. 170, 334-343 (2010).

27. Li, F. et al. Nebulin deficiency in adult muscle causes sarcomere defects and muscle-type-dependent changes in trophicity: novel insights in nemaline myopathy. Hum. Mol. Genet 24, 5219-5233 (2015).

28. Mijailovich, S. M. et al. Nebulin and titin modulate cross-bridge cycling and length-dependent calcium sensitivity. J. Gen. Physiol. 151, 680-704 (2019).

29. Li, F., Barton, E. R. \& Granzier, H. Deleting nebulin's C-terminus reveals its importance to sarcomeric structure and function and is sufficient to invoke nemaline myopathy. Hum. Mol. Genet. 28, 1709-1725 (2019).

30. Kiss, B. et al. Nebulin stiffens the thin filament and augments cross-bridge interaction in skeletal muscle. Proc. Natl Acad. Sci. USA 115, 10369-10374 (2018).

31. Kawai, M., Karam, T. S., Kolb, J., Wang, L. \& Granzier, H. L. Nebulin increases thin filament stiffness and force per cross-bridge in slow-twitch soleus muscle fibers. J. Gen. Physiol. 150, 1510-1522 (2018).

32. Bang, M. L. et al. Nebulin plays a direct role in promoting strong actin-myosin interactions. FASEB J. 23, 4117-4125 (2009).

33. Tonino, P. et al. Reduced myofibrillar connectivity and increased Z-disk width in nebulin-deficient skeletal muscle. J. Cell Sci. 123, 384-391 (2010).

34. Kiiski, K. J. et al. Dominantly inherited distal nemaline/cap myopathy caused by a large deletion in the nebulin gene. Neuromuscul. Disord. 29, 97-107 (2019).

35. Ottenheijm, C. A. et al. Deleting exon 55 from the nebulin gene induces severe muscle weakness in a mouse model for nemaline myopathy. Brain 136, 1718-1731 (2013).

36. Wallgren-Pettersson, C. et al. Distal myopathy caused by homozygous missense mutations in the nebulin gene. Brain 130, 1465-1476 (2007).

37. Pelin, K. et al. Nebulin mutations in autosomal recessive nemaline myopathy: an update. Neuromuscul. Disord. 12, 680-686 (2002).

38. Lehtokari, V. L. et al. Identification of 45 novel mutations in the nebulin gene associated with autosomal recessive nemaline myopathy. Hum. Mutat. 27, 946-956 (2006).
39. Marttila, M. et al. Nebulin interactions with actin and tropomyosin are altered by disease-causing mutations. Skelet. Muscle 4, 15 (2014).

40. Anderson, S. L. et al. Nemaline myopathy in the Ashkenazi Jewish population is caused by a deletion in the nebulin gene. Hum. Genet 115, 185-190 (2004).

41. Lehtokari, V. L. et al. The exon 55 deletion in the nebulin gene-one single founder mutation with world-wide occurrence. Neuromuscul. Disord. 19, 179-181 (2009).

42. Lawlor, M. W. et al. Novel mutations in NEB cause abnormal nebulin expression and markedly impaired muscle force generation in severe nemaline myopathy. Skelet. Muscle 1, 23 (2011).

43. Sewry, C. A. \& Wallgren-Pettersson, C. Myopathology in congenital myopathies. Neuropathol. Appl Neurobiol. 43, 5-23 (2017).

44. de Winter, J. M. \& Ottenheijm, C. A. C. Sarcomere dysfunction in nemaline myopathy. J. Neuromuscul. Dis. 4, 99-113 (2017).

45. Jungbluth, H. et al. Congenital myopathies: disorders of excitation-contraction coupling and muscle contraction. Nat. Rev. Neurol. 14, 151-167 (2018).

46. Ottenheijm, C. A. et al. Thin filament length dysregulation contributes to muscle weakness in nemaline myopathy patients with nebulin deficiency. Hum. Mol. Genet. 18, 2359-2369 (2009).

47. Winter, J. M. et al. Mutation-specific effects on thin filament length in thin filament myopathy. Ann. Neurol. 79, 959-969 (2016).

48. Fowler, V. M., Dominguez, R. Tropomodulins and leiomodins: actin pointed end caps and nucleators in muscles. Biophys. J. 112, 1742-1760 (2017).

49. Granzier, H. L. \& Labeit, S. Titin and its associated proteins: the third myofilament system of the sarcomere. Adv. Protein Chem. 71, 89-119 (2005)

50. Edman, K. A. The velocity of unloaded shortening and its relation to sarcomere length and isometric force in vertebrate muscle fibres. J. Physiol. 291, 143-159 (1979).

51. Yamamoto, D. L. et al. The nebulin SH3 domain is dispensable for normal skeletal muscle structure but is required for effective active load bearing in mouse. J. Cell Sci. 126, 5477-5489 (2013).

52. Malfatti, E. et al. Muscle histopathology in nebulin-related nemaline myopathy: ultrastrastructural findings correlated to disease severity and genotype. Acta Neuropathol. Commun. 2, 44 (2014).

53. Laitila, J. et al. A nebulin super-repeat panel reveals stronger actin binding toward the ends of the super-repeat region. Muscle Nerve 59, 116-121 (2019).

54. Lukoyanova, N. et al. Each actin subunit has three nebulin binding sites: implications for steric blocking. Curr. Biol. 12, 383-388 (2002).

55. Volkmann, N., DeRosier, D., Matsudaira, P. \& Hanein, D. An atomic model of actin filaments cross-linked by fimbrin and its implications for bundle assembly and function. J. Cell Biol. 153, 947-956 (2001).

56. Bordas, J. et al. Extensibility and symmetry of actin filaments in contracting muscles. Biophys. J. 77, 3197-3207 (1999).

57. Millman, B. M. The filament lattice of striated muscle. Physiol. Rev. 78, 359-391 (1998).

58. Fukuda, N. \& Granzier, H. L. Titin/connectin-based modulation of the FrankStarling mechanism of the heart. J. Muscle Res Cell Motil. 26, 319-323 (2005).

59. Ottenheijm, C. A., Hidalgo, C., Rost, K., Gotthardt, M. \& Granzier, H. Altered contractility of skeletal muscle in mice deficient in titin's M-band region. J. Mol. Biol. 393, 10-26 (2009).

60. Labeit, S. et al. Modulation of muscle atrophy, fatigue and MLC phosphorylation by MuRF1 as indicated by hindlimb suspension studies on MuRF1-KO mice. J. Biomed. Biotechnol. 2010, 693741 (2010).

61. Burkholder, T. J., Fingado, B., Baron, S. \& Lieber, R. L. Relationship between muscle fiber types and sizes and muscle architectural properties in the mouse hindlimb. J. Morphol. 221, 177-190 (1994).

62. Tonino, P. et al. The giant protein titin regulates the length of the striated muscle thick filament. Nat. Commun. 8, 1041 (2017).

63. Warren, C. M., Krzesinski, P. R. \& Greaser, M. L. Vertical agarose gel electrophoresis and electroblotting of high-molecular-weight proteins. Electrophoresis 24, 1695-1702 (2003).

64. Labeit, S. et al. Expression of distinct classes of titin isoforms in striated and smooth muscles by alternative splicing, and their conserved interaction with filamins. J. Mol. Biol. 362, 664-681 (2006).

65. Lahmers, S., Wu, Y., Call, D. R., Labeit, S. \& Granzier, H. Developmental control of titin isoform expression and passive stiffness in fetal and neonatal myocardium. Circ. Res. 94, 505-513 (2004).

66. Smith, L. R. \& Barton, E. R. SMASH - semi-automatic muscle analysis using segmentation of histology: a MATLAB application. Skelet. Muscle 4, 21 (2014).

67. Meng, H. et al. Tissue triage and freezing for models of skeletal muscle disease J. Vis. Exp. https://doi.org/10.3791/51586 (2014).

68. Brenner, B. \& Eisenberg, E. Rate of force generation in muscle: correlation with actomyosin ATPase activity in solution. Proc. Natl Acad. Sci. USA 83, 3542-3546 (1986).

69. Ma, W. et al. Thick-filament extensibility in intact skeletal muscle. Biophys. J. 115, 1580-1588 (2018).

70. Fischetti, R. et al. The BioCAT undulator beamline 18ID: a facility for biological non-crystalline diffraction and X-ray absorption spectroscopy at the Advanced Photon Source. J. Synchrotron Radiat. 11, 399-405 (2004). 
71. Ma, W., Gong, H. \& Irving, T. Myosin Head Configurations in Resting and Contracting Murine Skeletal Muscle. Int. J. Mol. Sci. 19, https://doi.org/ 10.3390/ijms19092643 (2018).

72. Wakabayashi, K. et al. X-ray diffraction evidence for the extensibility of actin and myosin filaments during muscle contraction. Biophys. J. 67, 2422-2435 (1994).

\section{Acknowledgements}

We are grateful to Drs. Carina Wallgren-Pettersson and Katarina Pelin (the Folkhälsan Institute of Genetics, University of Helsinki, Finland) for advice in selecting the missense mutation. This work was supported by grants from A Foundation Building Strength, and National Institute of Arthritis and Musculoskeletal and Skin Disease grant

R01AR053897. This research used resources of the Advanced Photon Source, operated for the DOE Office of Science by Argonne National Laboratory under Contract No. DEAC02-06CH11357. This project was supported by grant 9 P41 GM103622 and 1S10OD018090-01 from the National Institute of General Medical Sciences of the National Institutes of Health.

\section{Author contributions}

J.L., W.M, T.I. and H.G. designed the study. J.L, W.M., F.L., Y.H., J.K., B.K., P.T., R.v.d.P., E.K., H.G., J.S. and Z.H. collected the data. J.L., W.M., F.L., J.K., B.K., P.T., E.K., H.G., T.I. and H.G analyzed the data. J.L, W.M., T.I. and H.G. interpreted the data and wrote the manuscripts. All authors revised, reviewed and approved of the final version of the manuscript.

\section{Competing interests}

The authors declare no competing interests.

\section{Additional information}

Supplementary information is available for this paper at https://doi.org/10.1038/s41467020-16526-9.

Correspondence and requests for materials should be addressed to H.G.

Peer review information Nature Communications thanks Satoru Noguchi and the other, anonymous, reviewer(s) for their contribution to the peer review of this work. Peer reviewer reports are available.

Reprints and permission information is available at http://www.nature.com/reprints

Publisher's note Springer Nature remains neutral with regard to jurisdictional claims in published maps and institutional affiliations.

\section{(c) (i)}

Open Access This article is licensed under a Creative Commons Attribution 4.0 International License, which permits use, sharing, adaptation, distribution and reproduction in any medium or format, as long as you give appropriate credit to the original author(s) and the source, provide a link to the Creative Commons license, and indicate if changes were made. The images or other third party material in this article are included in the article's Creative Commons license, unless indicated otherwise in a credit line to the material. If material is not included in the article's Creative Commons license and your intended use is not permitted by statutory regulation or exceeds the permitted use, you will need to obtain permission directly from the copyright holder. To view a copy of this license, visit http://creativecommons.org/ licenses/by/4.0/.

(C) The Author(s) 2020 